\title{
Extension of the Poincaré Symmetry and Its Field Theoretical Implementation
}

\author{
Adrian TANAS $\breve{A}$
}

Laboratoire MIA, Faculté de Sciences et Techniques, Université de Haute Alsace, 4 rue des Frères Lumière, 68093 Mulhouse Cedex, France

E-mail: adrian.tanasa@ens-lyon.org

Received October 31, 2005, in final form April 28, 2006; Published online May 29, 2006

Original article is available at http://www.emis.de/journals/SIGMA/2006/Paper056/

\begin{abstract}
We define a new algebraic extension of the Poincaré symmetry; this algebra is used to implement a field theoretical model. Free Lagrangians are explicitly constructed; several discussions regarding degrees of freedom, compatibility with Abelian gauge invariance etc. are done. Finally we analyse the possibilities of interaction terms for this model.
\end{abstract}

Key words: extensions of the Poincaré algebra; field theory; algebraic methods; Lie (super)algebras; gauge symmetry

2000 Mathematics Subject Classification: 81T60; 17B99

\section{Introduction and motivation}

The notion of symmetry has always been playing a key role in fundamental particle physics. The conventional way to represent symmetries is, from a mathematical point of view, the notion of Lie algebra. Indeed, placing oneself within the framework of the Standard Model and using Lie algebras to group the symmetries of Nature, one has several powerful no-go theorems.

One can mention here the O'Raifeartaigh theorem [1] which further led to the ColemanMandula theorem [2]. Accepting the assumptions of the interacting relativistic quantum field theory $(\mathrm{QFT})^{1}$, the theorem states what are possible symmetries of Nature. One assumes that the symmetry group contains the Poincaré group as a subgroup, that the $S$-matrix is based on the local, interacting relativistic quantum field theory in 4 dimensions (thus one has e.g. analytic dependence of the center-of-mass energy $s$ and invariant momentum transfer $t$ of the elasticscattering amplitude); that there are only a finite-number of different particles associated with one-particle states of given mass and that there is energy gap between vacuum and one-particle states. The theorem then states that the demanded symmetry group is the direct product of an internal symmetry group and the Poincaré group. These internal symmetry transformations act only on particle-type indices and have no matrix elements between particles of different four-momentum or different spin.

However, a crucial hypothesis of the Coleman-Mandula theorem (completely natural at that moment) is utilisation, at infinitesimal level, of the notion of Lie algebras to group symmetries. It is exactly on utilisation of this hypothesis that we will speculate here.

Independently of all this, it is well-known that particle physics today needs to go beyond its Standard Model. One of the most appealing candidates for New Physics is supersymmetry (SUSY). SUSY extends the Poincaré symmetries by using a larger class of algebraic structures,

\footnotetext{
${ }^{1}$ An interested reader can consult for this purpose e.g. the book [3] of J. Lopuszanski. Note also that one can recall here the axiomatic approach to QFT (for example, the Wightman formalism [4]).
} 
Lie superalgebras. The Coleman-Mandula no-go theorem is therefore not contradicted. Moreover, a somewhat analogous no-go theorem exists, the Haag-Lopuszanski-Sohnius theorem [5] which states that, within the same framework of interacting relativistic QFT, the only Lie superalgebras extending the Poincaré symmetries are the SUSY extensions. Analogously to the Coleman-Mandula theorem, we put the emphasis here on the fact that this theorem uses Lie superalgebras to group symmetries.

Indeed, one may raise the question of using other type of algebraic structure to group symmetries. Here we review the use of a specific algebraic extension of the Poincaré algebra (introduced in a more formal framework in $[6,7])$ to the construction of a field theoretic model. The model treated here was first introduced in by N. Mohammedi et. al. in [8] and further developed by G. Moultaka et. al. in [9].

Let us also mention that a different algebraic structure, Lie parasuperalgebras [10], gave birth to a different field theoretic model called parasupersymmetry, which was developed by J. Beckers and N. Debergh in [11]. In relation to this subject note that parasupersymmetry was introduced in a non-equivalent way by V.A. Rubakov and V.P. Spiridonov in [12]. Furthermore, fractional supersymmetry was also analysed as a quantum mechanics model by a series of authors in $[13,14,15,16,17,18,19,20]$. Let us recall that a different approach was given by R. Kerner in [21]. Finally let us also mention that mathematical literature offers another example of an exotic algebraic structure, $n$-Lie algebras [22].

The physical motivation of such an approach is two-sided. First, the aim would be to develop a self-coherent model which may offer answers to some of today's open questions of fundamental physics via the use of new types of symmetries. However, if this is proven not to be the case, then such an approach should lead to enforcement of the existing no-go theorems.

The attempt presented here shows that there are considerable difficulties for constructing such a model. Therefore, as stated above, one may see this type of approach as a first step towards enforcement of no-go theorems, in the sense that stronger no-go theorems, which use also other types of algebraic structures (in this case cubic ones), might be obtained.

This review is organised as follows. In the Section 2 we give the general algebraic setting for this approach. In the Section 3 several comments are made on the foundation of this type of exotic approach. Connection with other constructions are discussed. In the Section 4 we construct bosonic multiplets associated to this structure. In the Section 5 a free theory is obtained. In the Section 6 we study the possibilities of interaction within the bosonic multiplets obtained previously. Finally, some technical details are given in the Appendix.

Let us also notice here that this review is a continuation of [23].

\section{Underlying algebraic structure}

In this section we give definitions for the algebraic setting used; general aspects are briefly discussed (for more details one may refer to $[6,7]$ and $[24]$ ).

In $[6,7]$, a complex Lie algebra of order $F\left(F \in \mathbb{N}^{*}\right)$ is defined as a $\mathbb{Z}_{F}$-graded $\mathbb{C}$-vector space $\mathfrak{g}=\mathfrak{g}_{0} \oplus \mathfrak{g}_{1} \oplus \mathfrak{g}_{2} \oplus \cdots \oplus \mathfrak{g}_{F-1}$ satisfying the following conditions:

1. $\mathfrak{g}_{0}$ is a complex Lie algebra.

2. For all $i \in\{1, \ldots, F-1\}, \mathfrak{g}_{i}$ is a representation of $\mathfrak{g}_{0}$.

3. For all $i=1, \ldots, F-1$ there exists an $F$-linear, $\mathfrak{g}_{0}$-equivariant map

$$
\mu_{i}: \mathcal{S}^{F}\left(\mathfrak{g}_{i}\right) \rightarrow \mathfrak{g}_{0}
$$

where $\mathcal{S}^{F}\left(\mathfrak{g}_{i}\right)$ denotes the $F$-fold symmetric product of $\mathfrak{g}_{i}$, satisfying the following (Jacobi) 
identity:

$$
\sum_{j=1}^{F+1}\left[Y_{j}, \mu_{i}\left(Y_{1}, \ldots, Y_{j-1}, Y_{j+1}, \ldots, Y_{F+1}\right)\right]=0
$$

for any $i=1, \ldots, F-1$ and for all $Y_{j} \in \mathfrak{g}_{i}, j=1, \ldots, F+1$.

Note that if $F=1$, by definition $\mathfrak{g}=\mathfrak{g}_{0}$ and a Lie algebra of order 1 is a Lie algebra. If $F=2$, then $\mathfrak{g}$ is a Lie superalgebra. In this sense, Lie algebras of order $F$ appear as some kind of generalisations of Lie algebras and superalgebras.

Note that, by definition, the following Jacobi identities are satisfied:

(i) For any $X, X^{\prime}, X^{\prime \prime} \in \mathfrak{g}_{0}$,

$$
\left[\left[X, X^{\prime}\right], X^{\prime \prime}\right]+\left[\left[X^{\prime}, X^{\prime \prime}\right], X\right]+\left[\left[X^{\prime \prime}, X\right], X^{\prime}\right]=0 .
$$

This relation expresses the fact that $\mathfrak{g}_{0}$ is a Lie algebra.

(ii) For any $X, X^{\prime} \in \mathfrak{g}_{0}$ and $Y \in \mathfrak{g}_{i}, i=1, \ldots, F-1$,

$$
\left[\left[X, X^{\prime}\right], Y\right]+\left[\left[X^{\prime}, Y\right], X\right]+\left[[Y, X], X^{\prime}\right]=0
$$

since $\mathfrak{g}_{i}$ is a representation of $\mathfrak{g}_{0}$.

(iii) For $X \in \mathfrak{g}_{0}$ and $Y_{j} \in \mathfrak{g}_{i}, j=1, \ldots, F, i=1, \ldots, F-1$

$$
\left[X, \mu_{i}\left(Y_{1}, \ldots, Y_{F}\right)\right]=\mu_{i}\left(\left[X, Y_{1}\right], \ldots, Y_{F}\right)+\cdots+\mu_{i}\left(Y_{1}, \ldots,\left[X, Y_{F}\right]\right)
$$

(results from the $\mathfrak{g}_{0}$-equivariance of the map $\mu_{i}$ ).

(iv) For all $Y_{j} \in \mathfrak{g}_{i}, j=1, \ldots, F+1, i=1, \ldots, F-1$,

$$
\sum_{j=1}^{F+1}\left[Y_{j}, \mu_{i}\left(Y_{1}, \ldots, Y_{j-1}, Y_{j+1}, \ldots, Y_{F+1}\right)\right]=0,
$$

which corresponds to condition 3 of the definition.

Recall here that in the case of Lie (super)algebras Jacobi identities are included as a necessary element which is related to the associativity of the corresponding Lie groups. However, in our case, even though one has corresponding Jacobi identities (see above), one does not know a corresponding exponentiation to obtain some "group".

One important thing to notice here is that, if $\mathfrak{g}_{0} \oplus \mathfrak{g}_{1} \oplus \cdots \oplus \mathfrak{g}_{F-1}$ is a Lie algebra of order $F$, with $F>1$, for any $i=1, \ldots, F-1$, the vector spaces $\mathfrak{g}_{0} \oplus \mathfrak{g}_{i}$ is also Lie algebra of order $F$. The particular algebraic extension we use here is of this type and considers the case $F=3$; we denote the map $\mu$ by the 3 -entries bracket $\{\cdot, \cdot, \cdot\}$; the algebra writes:

$$
\begin{aligned}
& {\left[L_{m n}, L_{p q}\right]=i\left(\eta_{n q} L_{p m}-\eta_{m q} L_{p n}+\eta_{n p} L_{m q}-\eta_{m p} L_{n q}\right),} \\
& {\left[L_{m n}, P_{p}\right]=i\left(\eta_{n p} P_{m}-\eta_{m p} P_{n}\right),} \\
& {\left[L_{m n}, V_{p}\right]=i\left(\eta_{n p} V_{m}-\eta_{m p} V_{n}\right), \quad\left[P_{m}, V_{n}\right]=0,} \\
& \left\{V_{m}, V_{n}, V_{r}\right\}=\eta_{m n} P_{r}+\eta_{m r} P_{n}+\eta_{r n} P_{m},
\end{aligned}
$$

where

$$
\left\{V_{m}, V_{n}, V_{r}\right\}=V_{m} V_{n} V_{r}+V_{m} V_{r} V_{n}+V_{n} V_{m} V_{r}+V_{n} V_{r} V_{m}+V_{r} V_{m} V_{n}+V_{r} V_{n} V_{m}
$$


stands for the symmetric product of order $3, L_{m n}$ are the usual generators of the Lorentz algebra $\mathfrak{s o}(1,3), P_{m}$ are the momentums $(m, n=0, \ldots, 3)$ and $\eta_{m n}=\operatorname{diag}(1,-1,-1,-1)$ is the Minkowski metric.

Note that, from the point of view of fundamental physics, there is no special in this value of $F$; this approach attempts, as was already stated before, to explore the possibility of constructing a field theoretical model based on cubic symmetries.

Comparing to the SUSY extension one can already make a certain number of comments. As already noticed, by using a different algebraic structure a construction of this type evades a priori the hypothesis of no-go theorems. Moreover, one can notice from the second line of (1) that the 3-charges $V$ lie in the vector representation of the Lorentz algebra and not in the spinor representation, as was the case for SUSY. This will have as a first consequence the fact that the multiplets to be obtained later will be either bosonic or fermionic (one will not have statistics-mixing multiplets, as is the case for SUSY).

Another thing to notice is the following: if the supercharges $Q$ are referred to as "square roots of translations" (since $Q^{2} \propto P$, see for example [25]), we can speak of the generators $V$ as some kind of "cubic roots of translations" ( since $V^{3} \propto P$ ).

\section{Comments on the foundation of this approach; connection with other constructions}

We have thus seen in the previous sections the motivation and the basis of the foundation of this approach. In this section we argue more thoroughly on some implications of such a construction when reporting it to the usual concepts of QFT. We start by having a closer look to the representations of the complexified Poincaré algebra. Then, the first subsection makes a formal connection between the generators $V$ and the the supercharges $Q$ of SUSY. The second subsection refers to the assumption of analytic dependence of a field theoretic model.

\subsection{Representations of dimension 4 of the Poincaré algebra}

In studying different classifications of the extensions of Poincaré algebra, a key role is played by the study of its representations. We present here such results for 4-dimensional representations, other results also to be found in [24].

Notice here that, when considering both our extension and the SUSY extension ${ }^{2}$, one uses representations of the Poincaré algebra of dimension 4. We now point out what are all these 4-dimensional representations.

For this, consider the complexified Poincaré algebra. It is well-known that its semi-simple part is isomorphic to $\mathfrak{s l}(2) \oplus \mathfrak{s l}(2)$. This can be seen explicitly by performing the following change of basis in the Lorentz algebra:

$$
\begin{aligned}
& U_{1}=i M_{01}-M_{23}, \quad U_{2}=-\frac{1}{2}\left(M_{02}-M_{12}+i M_{03}-i M_{13}\right), \\
& U_{3}=\frac{1}{2}\left(M_{02}+M_{12}-i M_{03}-i M_{13}\right), \\
& W_{1}=i M_{01}+M_{23}, \quad W_{2}=-\frac{1}{2}\left(M_{02}-M_{12}-i M_{03}+i M_{13}\right), \\
& W_{3}=\frac{1}{2}\left(M_{02}+M_{12}+i M_{03}+i M_{13}\right) .
\end{aligned}
$$

\footnotetext{
${ }^{2}$ Obviously, we refer here to the simplest SUSY extension, $N=1$ and with no central charges (see for example $[25])$.
} 
The semi-simple part writes

$$
\begin{array}{ll}
{\left[U_{1}, U_{2}\right]=-2 U_{2},} & {\left[W_{1}, W_{2}\right]=-2 W_{2},} \\
{\left[U_{1}, U_{3}\right]=2 U_{3},} & {\left[W_{1}, W_{3}\right]=2 W_{3},} \\
{\left[U_{3}, U_{2}\right]=U_{1},} & {\left[W_{3}, W_{2}\right]=W_{1} .}
\end{array}
$$

The basis (3) is more handy when one confront the problem of writing down all the representations of the complexified Poincaré algebra. (Indeed, then one can make direct use of the highest weight representations of $\mathfrak{s l}(2)$.)

Let us denote now the spin $j$ representations of $\mathfrak{s l}(2)$ by $\mathcal{D}_{j}$. Furthermore, denote by $\mathcal{D}_{\left(j_{1}, j_{2}\right)}$ the representation of $\mathfrak{s l}(2) \oplus \mathfrak{s l}(2)$ of spin $j_{1}$ with respect to the first copy of $\mathfrak{s l}(2)$ and of spin $j_{2}$ with respect to the second copy of $\mathfrak{s l}(2)$, and thus of dimension $\left(2 j_{1}+1\right)\left(2 j_{2}+2\right)$.

Then all the 4-dimensional representations of $\mathfrak{s l}(2) \oplus \mathfrak{s l}(2)$ are:

$$
\mathcal{D}_{\left(\frac{3}{2}, 0\right)}, \mathcal{D}_{(1,0)} \oplus \mathcal{D}_{(0,0)}, \mathcal{D}_{\left(\frac{1}{2}, \frac{1}{2}\right)}, \mathcal{D}_{\left(\frac{1}{2}, 0\right)} \oplus \mathcal{D}_{\left(0, \frac{1}{2}\right)}, \mathcal{D}_{\left(\frac{1}{2}, 0\right)} \oplus \mathcal{D}_{(0,0)}^{2}, \mathcal{D}_{(0,0)}^{4} .
$$

Notice that the third representation in this list corresponds to the vector representation of the Poincaré algebra, the representation where the momentums $P_{m}$ also lie. Moreover, it is also the representation in which the vector generators $V_{m}$ of (1) lie. The fourth representation in this list is a spinor representation, and the supercharges $Q$ of the SUSY extensions lie here.

Consider now the 4 pairs of weights $\alpha_{i}, i=1, \ldots, 4$ (with respect to two copies of $\mathfrak{s l}(2)$ ) of the 4 generators of the representation considered here. The list (4) leads to the following table of weights:

\begin{tabular}{|c|c|c|c|}
\hline$\alpha_{1}$ & $\alpha_{2}$ & $\alpha_{3}$ & $\alpha_{4}$ \\
\hline$(-3,0)$ & $(-1,0)$ & $(1,0)$ & $(3,0)$ \\
\hline$(-2,0)$ & $(0,0)$ & $(2,0)$ & $(0,0)$ \\
\hline$(1,-1)$ & $(-1,1)$ & $(1,1)$ & $(-1,-1)$ \\
\hline$(1,0)$ & $(-1,0)$ & $(1,0)$ & $(-1,0)$ \\
\hline$(1,0)$ & $(-1,0)$ & $(0,0)$ & $(0,0)$ \\
\hline$(0,0)$ & $(0,0)$ & $(0,0)$ & $(0,0)$ \\
\hline
\end{tabular}

\subsection{Formal connection with the SUSY supercharges}

We make here a formal connection between the bracket for the generators $V$ (bracket defined in equation (2)) and the usual anticommutator of two supercharges $Q$. This approach is inspired by the fact that, as already noticed in Section 2, the SUSY supercharges $Q$ are square roots of translations and the generators $V$ are cubic roots of translations. Hence one may think of a possible connection $V^{3} \propto P \propto Q^{2}$. More exactly, recall from the 3SUSY algebra (1) the relation

$$
\left\{V_{m}, V_{n}, V_{r}\right\}=\eta_{m n} P_{r}+\eta_{m r} P_{n}+\eta_{r n} P_{m} .
$$

The SUSY algebra (with the conventions of [25]) includes the relation:

$$
\left\{Q_{\alpha}, \bar{Q}_{\dot{\beta}}\right\}=2\left(\sigma^{m}\right)_{\alpha \dot{\beta}} P_{m}
$$

(with $\alpha, \dot{\beta}=1,2$ ). Hence one may calculate some connection between the 3 -entries bracket (5) and the anticommutator (6). Writing explicitly the SUSY algebra anticommutators (6), one can then express any momentum $P_{m}$ as a function of them; these expressions are then inserted in (5) thus leading to

$$
\left\{V_{m}, V_{n}, V_{r}\right\}=\left(\sigma_{m n r}\right)^{\alpha \dot{\beta}}\left\{Q_{\alpha}, \bar{Q}_{\dot{\beta}}\right\},
$$

where the matrices $\sigma_{m n r}$ have the explicit form (60). 
The purpose of such connection with the SUSY supercharges would be to acquire some information on what would be the "allowed" possibilities for a vector generator which extends the Poincaré symmetry. However, this does not seem to be the case here, because in all generality (that is not considering only Lie superalgebras) the SUSY supercharges $Q$ are not the only fermionic generators to extend the Poincaré symmetries. Indeed, this is the case, as proved by the Haag-Lopuszanski-Sohnius theorem, but only within the framework of Lie superalgebras. Considering other type of algebraic structures, other type of fermionic generators may a priori lead to non-trivial extensions and hence relations of type (7) could be obtained.

\subsection{Connection with other constructions}

Already in the introduction we have mentioned other extensions of the Poincaré symmetry. Some other constructions are now referred to.

In [26], G. Grignani et. al. propose a model for planar $P, T$-invariant fermions. In this model, a hidden $N=3$ SUSY leads to a non-standard super-extension of the $(2+1)$-dimensional Poincaré group. In [27], K.S. Nirov and M.S. Plyushchay investigate hidden symmetries of a $P, T$ invariant system of topologically massive $U(1)$ gauge fields. This system realizes an irreducible representation of a non-standard super-extension of the $(2+1)$-dimensional Poincaré group. The non-standard behaviour means that the anticommutator of corresponding supercharges results in an operator different from the Hamiltonian.

In the context of parabosonic systems, in [28], M.S. Plyushchay founds a hidden polynomial SUSY. One may wonder whether or not a relation between this structure and the structure investigated in this review can be established. This seems to be unlikely, since, even if nonlinear, the algebraic structure of [28] is a superalgebra, which is not the case here. Thus, the two structure are already different at the level of the definition of the algebraic structure. However, one may further address the issue of a possible formal connection, like the one established in the previous subsection for the case with the SUSY algebra. This still does not seem to be the case for the non-linear superalgebra appearing in [28], because one cannot easily obtain the momentum as function of the anticommutators, as was done in Appendix A for the case of the SUSY algebra.

Another interesting issue is related to the construction of [29], where a relativistic wave equation involving the cubic root of the Klein-Gordon operator is defined. The equation considered here can be related to fractional supersymmetry in the following sense: one considers localised 1-dimensional fractional supersymmetry, fractional supergravity; quantising this model one obtains this new type of wave equation (see [16]).

\subsection{The assumption of analicity}

We have seen in section 1 that one of the assumptions of the Coleman-Mandula theorem was the analytic dependence of the elastic scattering amplitude on the momentum and spin variables. As S. Coleman and J. Mandula say in their original paper [2], the naturalness of this assumption being above any doubt, being "something that most physicist believe to be a property of the real world". In this subsection we will look to this issue in more detail and give an illustration of why additional exotic symmetries in the Standard Model frame would violate this assumption. We do this explicitly on a simple example of two-body scattering. We then discuss what this becomes for the case of SUSY and of the algebra (1). This short discussion is drawn upon E. Witten's analyse in [30].

In the simplest case of two-particle scattering, considering that the momentum and angular momentum are the only conserved charges, one has the cross section depending only on the scattering angle $\theta$. 
Let us now assume that one has an additional conserved charge, say a symmetric, traceless tensor $Z_{m n}$, which closes with the rest of the Poincaré generators by commutation relations. These commutation relations are not trivial and hence $Z_{m n}$ has no trivial matrix elements between particles of different four-momentum and spin.

For simplicity, we consider here only spinless particle states. By Lorentz invariance, one takes for the matrix element of $Z_{m n}$ in a one-particle state of momentum $p$

$$
\left\langle p\left|Z_{m n}\right| p\right\rangle=p_{m} p_{n}-\frac{1}{4} \eta_{m n} p^{2} .
$$

(The new matrix element is expressed with the help of the momentum four-vector.) Now, for the two-particle scattering above, assume that the matrix element in the two-particle state $\left|p_{1} p_{2}\right\rangle$ is the sum of the matrix elements in the states $\left|p_{1}\right\rangle$ and $\left|p_{2}\right\rangle$. Hence, the conservation of $Z$, $\left\langle p_{1} p_{2}\left|Z_{m n}\right| p_{1} p_{2}\right\rangle=\left\langle p_{1}^{\prime} p_{2}^{\prime}\left|Z_{m n}\right| p_{1}^{\prime} p_{2}^{\prime}\right\rangle$, leads to $p_{1 m} p_{1 n}+p_{2 m} p_{2 n}=p_{1 m}^{\prime} p_{1 n}^{\prime}+p_{2 m}^{\prime} p_{2 n}^{\prime}$. This is a supplementary conservation law, which can be satisfied only for $\theta=0$, and hence the analytic dependence is lost.

If one considers now the case of SUSY, one cannot make the same reasoning. Indeed, since $Q$ is a spinor, one cannot construct a matrix element $\left\langle p\left|Q_{\alpha}\right| p\right\rangle$ (the analogous of (8)) with the help of only momentum and spin variables.

To conclude this section, let us now shortly discuss what would this become for the case of the construction (1). As we have already mentioned the additional symmetries $V_{m}$ are Lorentz vectors and close within a structure of Lie algebra of order 3 . Hence, one can a priori construct a matrix element like, for example $\left\langle p\left|V_{m}\right| p\right\rangle=C p_{m}$, which will lead to a certain constraint. This gives rise to a new, exotic conservation law which, as above, would contradict analytic dependence; the theory constructed upon in 4 dimensions would have to be non-interacting.

This short remark is an indication towards conclusion on impossibility of interactions for the model discussed in this review. This goes along the result we prove in Section 6 .

\section{Representations of the algebra, bosonic multiplets}

We have so far introduced the algebra (1) and argued on some of the foundations of this type of exotic approach. In this section we start construction of the field theoretic model as follows. Irreducible matrix representations are exhibited. These lead to different type of multiplets (bosonic or fermionic) amongst which we consider here the bosonic ones. Transformation laws of these bosonic fields are then obtained. We then give some technical properties of these multiplets, the properties that will prove to be useful for the sequel. Before ending this section we stop for a moment from the implementation of our field theoretical moment and we make some comments regarding the relation with the spin-statistics theorem.

\subsection{Irreducible representations}

The algebra (1) has two inequivalent 6-dimensional representations

$$
V_{+m}=\left(\begin{array}{ccc}
0 & \Lambda^{1 / 3} \sigma_{m} & 0 \\
0 & 0 & \Lambda^{1 / 3} \bar{\sigma}_{m} \\
\Lambda^{-2 / 3} \partial_{m} & 0 & 0
\end{array}\right), \quad V_{-m}=\left(\begin{array}{ccc}
0 & \Lambda^{1 / 3} \bar{\sigma}_{m} & 0 \\
0 & 0 & \Lambda^{1 / 3} \sigma_{m} \\
\Lambda^{-2 / 3} \partial_{m} & 0 & 0
\end{array}\right)
$$

with $\sigma^{m}=\left(\sigma^{0}=1, \sigma^{i}\right)$, and $\bar{\sigma}^{m}=\left(\bar{\sigma}^{0}=1,-\sigma^{i}\right), \sigma^{i}$ the Pauli matrices and $\Lambda$ a parameter with mass dimension that we take equal to 1 (in appropriate units). For details on the manner this representation has been obtained, one can refer to [8]. These two representations are referred to as conjugated to each other and they will give rise to different types of multiplets, as we will see later on. 
One may also notice here that these representations are not proven to be the only irreducible representations. Thus, if other representations exist then they may lead also to physics results.

\section{$4.2 \quad$ Multiplets}

The matrices $V_{+}$and resp. $V_{-}(9)$ act on the triplets of Weyl spinors $\Psi_{+}$and resp. $\Psi_{-}$,

$$
\Psi_{+}=\left(\begin{array}{c}
\psi_{1+} \\
\psi_{2-} \\
\psi_{3+}
\end{array}\right) \quad \text { or } \quad \Psi_{-}=\left(\begin{array}{c}
\psi_{1-} \\
\psi_{2+} \\
\psi_{3-}
\end{array}\right)
$$

where $\psi_{1+}$ is a left-handed (LH) 2-component Weyl spinor, $\bar{\psi}_{2-}$ is a right-handed (RH) 2-component Weyl spinor etc.

The transformation law associated to this type of new symmetry writes

$$
\delta_{v} \Psi_{ \pm}=v^{m} V_{ \pm m} \Psi_{ \pm},
$$

where $v$ is the transformation parameter.

The representation space of the algebra (1) is constituted of the states generated by $\Psi$. A very interesting remark to be made here is that this representation space has a $\mathbb{Z}_{3}$-graded structure (the representation space for the SUSY algebra having a similar $\mathbb{Z}_{2}$-graded structure). Technically, this result comes from the dimension of the irreducible representation, $6=3 \cdot 2$ : the 6-dimensional matrices $V_{m}$ act on 3 copies of Weyl spinors (which are each of them 2dimensional).

In further use, we call the states of $\psi_{1}$ states of gradation -1 , the states of $\psi_{2}$ states of gradation 0 and the states of $\psi_{3}$ states of gradation 1.

One has further similitude with SUSY, in the sense already mentioned of "cubic roots of translations": one has $\psi_{1} \rightarrow \psi_{2} \rightarrow \psi_{3} \rightarrow \psi_{1}$ (that is, acting with the generator $V$ on a state of gradation -1 one has a state of gradation 0 , acting again with a generator $V$ one has a state of gradation 1 and finally, acting one more time with a generator $V$, one has a state of gradation -1). One could have reached the same final state just by acting with some translation generator on the initial state (recall that a similar phenomena happened in the case of SUSY, see for example [25]). Some comments regarding this issue and the relation to the spin-statistics theorem are made in the last subsection of this section.

Taking now into consideration the vacuum, a singlet for this new symmetry, one has to specify in which representation of the Lorentz algebra it lies. Considering it in the trivial representation of the Lorentz algebra, equation (10) leads directly to two fermionic multiplets. We will not be concerned here with these fermionic multiplets; they have been treated in [8] where a nonconventional kinetic Lagrangian was obtained.

Another possibility for the vacuum, a Lorentz vector, was also treated in [8] leading to the same type of results.

However, considering now the vacuum lying in the spinor representation of the Lorentz algebra, one obtains bosonic multiplets. The vacuum can be a LH or RH Weyl spinor, $\Omega_{+}$and $\Omega_{-}$. Therefore one has four possibilities $\boldsymbol{\Xi}_{ \pm \pm}$for a tensor product $\Psi_{ \pm} \otimes \Omega$, with $\Psi_{ \pm}$given in (10)

$$
\begin{aligned}
& \Xi_{++}=\Psi_{+} \otimes \Omega_{+}=\left(\begin{array}{l}
\Xi_{1++} \\
\Xi_{2-+} \\
\Xi_{3++}
\end{array}\right), \quad \Xi_{--}=\Psi_{-} \otimes \Omega_{-}=\left(\begin{array}{c}
\Xi_{1--} \\
\Xi_{2+-} \\
\Xi_{3--}
\end{array}\right), \\
& \Xi_{-+}=\Psi_{-} \otimes \Omega_{+}=\left(\begin{array}{l}
\Xi_{1-+} \\
\Xi_{2++} \\
\Xi_{3-+}
\end{array}\right), \quad \Xi_{+-}=\Psi_{+} \otimes \Omega_{-}=\left(\begin{array}{c}
\Xi_{1+-} \\
\Xi_{2--} \\
\Xi_{3+-}
\end{array}\right) .
\end{aligned}
$$


The following step is decomposition of these products of spinors on $p$-forms. For the case of $\boldsymbol{\Xi}_{++}$, defined in equation (12)), this writes (see [23] for technical details)

$$
\Xi_{1++}=\varphi+\frac{1}{4} B_{m n} \sigma^{m n}, \quad \Xi_{2-+}=\tilde{A}_{m} \bar{\sigma}^{m}, \quad \Xi_{3++}=\tilde{\tilde{\varphi}}+\frac{1}{4} \tilde{\tilde{B}}_{m n} \sigma^{m n}
$$

where we denote by $\varphi, \tilde{\tilde{\varphi}}$ two scalar fields, $\tilde{A}_{m}$ a vector and $B_{m n}, \tilde{\tilde{B}}_{m n}$ two self-dual 2-forms.

Applying this analysis for the four product of spinors (12) leads to the four multiplets $\boldsymbol{\Xi}_{ \pm \pm}$ with the following field content

$$
\begin{aligned}
& \boldsymbol{\Xi}_{++}=\left(\begin{array}{c}
\varphi, B_{m n} \\
\tilde{A}_{m} \\
\tilde{\tilde{\varphi}}, \tilde{B}_{m n}
\end{array}\right), \quad \boldsymbol{\Xi}_{+-}=\left(\begin{array}{c}
A_{m}^{\prime} \\
\tilde{\varphi}^{\prime}, \tilde{B}_{m n}^{\prime} \\
\tilde{\tilde{A}}_{m}^{\prime}
\end{array}\right), \\
& \boldsymbol{\Xi}_{--}=\left(\begin{array}{c}
\varphi^{\prime}, B_{m n}^{\prime} \\
\tilde{A}_{m}^{\prime} \\
\tilde{\tilde{\varphi}}^{\prime}, \tilde{\tilde{B}}_{m n}^{\prime}
\end{array}\right),
\end{aligned}
$$

where $\varphi, \tilde{\tilde{\varphi}}, \varphi^{\prime}, \tilde{\tilde{\varphi}}^{\prime}, \tilde{\varphi}, \tilde{\varphi}^{\prime}$ are scalars fields, $\tilde{A}, \tilde{A}^{\prime}, A, \tilde{\tilde{A}}, A^{\prime}, \tilde{\tilde{A}}^{\prime}$ are vector fields, $B, \tilde{B}, \tilde{\tilde{B}}, B^{\prime}$, $\tilde{B}^{\prime}, \tilde{\tilde{B}}^{\prime}$ are 2 -forms. As we have mentioned above, these 2 -forms, namely $B, \tilde{B}, \tilde{\tilde{B}}$ are self-dual (i.e. ${ }^{*} B=i B$, where by ${ }^{*} B$ we mean the dual of $B$ ) and resp. $B^{\prime}, \tilde{B}^{\prime}, \tilde{\tilde{B}}^{\prime}$ are anti-self-dual (i.e. ${ }^{*} B^{\prime}=-i B^{\prime}$ ); thus these 2-forms must be complex. To have minimum field content, one takes $\boldsymbol{\Xi}_{++}=\boldsymbol{\Xi}_{--}^{*}$ and $\boldsymbol{\Xi}_{+-}=\boldsymbol{\Xi}_{-+}^{*}$ (that is $\varphi=\varphi^{*}, B=B^{*}$, etc.) We call the couples $\boldsymbol{\Xi}_{++}-\boldsymbol{\Xi}_{--}$, $\boldsymbol{\Xi}_{+-}-\boldsymbol{\Xi}_{-+}$conjugated multiplets and the couples $\boldsymbol{\Xi}_{++}-\boldsymbol{\Xi}_{+-}, \boldsymbol{\Xi}_{--}-\boldsymbol{\Xi}_{-+}$interlaced multiplets.

Following the convention defined earlier, one can say, for example for the multiplet $\boldsymbol{\Xi}_{++}$that the fields $\varphi, B$ are of gradation -1 , the field $\tilde{A}$ is of gradation 0 and the fields $\tilde{\varphi}$, $\tilde{\tilde{B}}$ are of gradation 1 .

At this level of our construction let us notice that in the same multiplet one has scalar and vector fields as well as 2-form. In SUSY models, the scalar fields combine with fermions making supermultiplets. Here, as noted before, since the generators $V$ lie in the vector representation of the Lorentz algebra, one has multiplets of the same statistics, that is either bosonic or fermionic multiplets. Later on in this section we obtain Lagrangians presenting explicit gauge fixation terms for the fields. Moreover, in Subsection 5.4, compatibility of this new type of symmetry with the Abelian gauge invariance is analysed. This means that, if one applies a gauge transformation to some multiplet, the same type of multiplet is obtained (this property being also present for SUSY models).

\subsection{Transformation laws of the fields}

The transformation laws of the fields are obtained from the transformation law (11), using the explicit form (9) of the matrices $V_{ \pm m}$ (see again [23] for detailed calculus). We give the explicit formulae obtained for the $\boldsymbol{\Xi}_{++}$multiplet, similar formulae being obtained for the other multiplets:

$$
\begin{aligned}
& \delta_{v} \varphi=v^{m} \tilde{A}_{m}, \quad \delta_{v} B_{m n}=-\left(v_{m} \tilde{A}_{n}-v_{n} \tilde{A}_{m}\right)+i \varepsilon_{m n q q} v^{p} \tilde{A}^{q} \\
& \delta_{v} \tilde{A}_{m}=\left(v_{m} \tilde{\tilde{\varphi}}+v^{n} \tilde{\tilde{B}}_{m n}\right), \quad \delta_{v} \tilde{\tilde{\varphi}}=v^{m} \partial_{m} \varphi, \quad \delta_{v} \tilde{\tilde{B}}_{m n}=v^{p} \partial_{p} B_{m n} .
\end{aligned}
$$

\subsection{Derivation of a multiplet}

We now obtain an interesting property of these multiplets, property which will be used when analysing compatibility of our model with Abelian gauge invariance (see Subsection 5.4) and when treating the possibilities of interaction (see Section 6). 
Let us denote from now on by $X_{[m n] \pm}$ the (anti-)self-dualisation of any second rank tensor $X_{m n}$, i.e. $X_{[m n]_{ \pm}}=X_{m n}-X_{n m} \mp i \varepsilon_{m n p q} X^{p q}$.

Let us now consider the fields of a $\boldsymbol{\Xi}_{+-}$multiplet, that is $A_{m}^{\prime} ; \tilde{\varphi}^{\prime}, \tilde{B}_{m n}^{\prime} ; \tilde{\tilde{A}}_{m}^{\prime}$ to construct a different type of multiplet using partial derivatives $\partial_{m}$. Thus, to construct a $\boldsymbol{\Xi}_{++}$multiplet, one has to have expressions for any field of the $\boldsymbol{\Xi}_{++}$multiplet. Saturating the Lorentz indices, respecting the $\mathbb{Z}_{3}$ gradation and the (anti-)self-dual character of the different 2-forms, one possible solution is

$$
\begin{aligned}
\mathcal{D} \boldsymbol{\Xi}_{+-} & =\left(\psi, \psi_{m n}, \tilde{\psi}_{m}, \tilde{\tilde{\psi}}, \tilde{\tilde{\psi}}_{m n}\right) \\
& \equiv\left(\partial_{m} A^{\prime m}, \partial_{[m} A_{n]_{+}}^{\prime} ; \partial_{m} \tilde{\varphi}^{\prime}+\partial^{n} \tilde{B}_{n m}^{\prime} ; \partial_{m} \tilde{\tilde{A}}^{\prime m}, \partial_{[m} \tilde{\tilde{A}}_{n]_{+}}^{\prime}\right) .
\end{aligned}
$$

The last thing for this set to form a $\boldsymbol{\Xi}_{++}$multiplet is that it transforms as requested by equation (15). This is checked by directly applying the transformations laws (15) on equation (16). Thus we have shown a mechanism to obtain a multiplet of a certain type (here $\boldsymbol{\Xi}_{++}$) by "deriving" a multiplet of another type (here $\boldsymbol{\Xi}_{+-}$). We call $\mathcal{D} \boldsymbol{\Xi}_{+-}$a derivative multiplet. One can actually define such a "derivation" for every 3SUSY multiplet $\boldsymbol{\Xi}_{ \pm \pm}$.

\subsection{The spin-statistics connection}

Before going further in developing this model, we would like to address here the legitimate question of the connection with the spin-statistics theorem, theorem which states that bosons obey the Bose-Einstein statistics and fermions obey the Fermi-Dirac statistics. So one may ask where does our model stay from this point of view?

The additional symmetries $V$ even though they lie in the vector representation of the Lorentz algebra do not close with classical (anti)commutation relations. Usually in physics literature (for example in the case of superalgebras), one denotes generators that close with commutators as bosonic generators and to generators that close with anticommutators as fermionic generators. Obviously this is not the case here: $V$ are neither bosonic nor fermionic generators (this is how our construction evades the no-go theorems and apparently, one of the prices to pay). Nevertheless, the physical fields $\varphi, A, B$ are bosons (and in the case of the fermionic multiplets in [8] they are fermions), thus obeying the conventional statistics. Technically, we got to this situation by decomposition on $p$-forms.

A connected aspect here is the following. In the SUSY case, one has a $\mathbb{Z}_{2}$-graded structure which, at the level of the representation space translates by a division of the representation space in two subspaces, a bosonic and a fermionic subspace. This interpretation is obviously lost here: we have now a $\mathbb{Z}_{3}$-graded representation space and we do not have a correspondence of these subspaces to some type of particle, as was the case for SUSY. However, as mentioned above, one finds the conventional types of fields at the level of the physical fields $\varphi, A, B$ etc.

\section{$5 \quad$ Free theory}

In this section we construct free Lagrangians invariant under the transformations (15); one thus obtains a new symmetry. From now on we denote the field strengths associated to the fields by $F_{m n}=\partial_{m} A_{n}-\partial_{n} A_{m}$ for any vector field $A_{m}$ and by $H_{m n p}=\partial_{m} B_{n p}+\partial_{p} B_{m n}+\partial_{n} B_{p m}$ for any 2 -form $B_{m n}$.

\subsection{Coupling between conjugated multiplets}

If we consider the quadratic couplings between conjugated multiplets, as denoted in Subsection 4.2 , one can construct two invariant Lagrangians, one for each pair $\boldsymbol{\Xi}_{++}-\boldsymbol{\Xi}_{--}$and 


$$
\begin{aligned}
\boldsymbol{\Xi}_{+-}-\boldsymbol{\Xi}_{-+} & \\
\mathcal{L}_{0}= & \mathcal{L}_{0}\left(\boldsymbol{\Xi}_{++}\right)+\mathcal{L}_{0}\left(\boldsymbol{\Xi}_{--}\right) \\
= & \partial_{m} \varphi \partial^{m} \tilde{\tilde{\varphi}}+\frac{1}{12} H_{m n p} \tilde{\tilde{H}}^{m n p}+\frac{1}{2}{ }^{\star} H_{m}{ }^{\star} \tilde{\tilde{H}}^{m}-\frac{1}{4} \tilde{F}_{m n} \tilde{F}^{m n}-\frac{1}{2}\left(\partial_{m} \tilde{A}^{m}\right)^{2} \\
& +\partial_{m} \varphi^{\prime} \partial^{m} \tilde{\tilde{\varphi}}^{\prime}+\frac{1}{12} H_{m n p}^{\prime} \tilde{\tilde{H}}^{\prime m n p}+\frac{1}{2}{ }^{\star} H_{m}^{\prime}{ }^{\star} \tilde{\tilde{H}}^{\prime m}-\frac{1}{4} \tilde{F}_{m n}^{\prime} \tilde{F}^{\prime m n}-\frac{1}{2}\left(\partial_{m} \tilde{A}^{\prime m}\right)^{2}
\end{aligned}
$$

and

$$
\begin{aligned}
\mathcal{L}^{\prime}= & \mathcal{L}_{0}\left(\boldsymbol{\Xi}_{-+}\right)+\mathcal{L}_{0}\left(\boldsymbol{\Xi}_{+-}\right) \\
= & \frac{1}{2} \partial_{m} \tilde{\varphi} \partial^{m} \tilde{\varphi}+\frac{1}{24} \tilde{H}_{m n p} \tilde{H}^{m n p}-\frac{1}{4}{ }^{\star} \tilde{H}_{m}{ }^{\star} \tilde{H}^{m}-\frac{1}{2} F_{m n} \tilde{\tilde{F}}^{m n}-\left(\partial_{m} A^{m}\right)\left(\partial_{n} \tilde{\tilde{A}}^{n}\right) \\
& +\frac{1}{2} \partial_{m} \tilde{\varphi}^{\prime} \partial^{m} \tilde{\varphi}^{\prime}+\frac{1}{24} \tilde{H}_{m n p}^{\prime} \tilde{H}^{\prime m n p}-\frac{1}{4}{ }^{\star} \tilde{H}_{m}^{\prime}{ }^{\star} \tilde{H}^{\prime m}-\frac{1}{2} F_{m n}^{\prime} \tilde{\tilde{F}}^{m n}-\left(\partial_{m} A^{\prime m}\right)\left(\partial_{n} \tilde{\tilde{A}}^{\prime n}\right) .
\end{aligned}
$$

We consider here $\mathcal{L}_{0}$, the analyse for $\mathcal{L}_{0}^{\prime}$ being identical. Here we have denoted ${ }^{*} H_{m}=$ $\frac{1}{6} \varepsilon_{m n p q} H^{n p q}=\partial^{n} B_{m n}$ the dual of the field strength $H$. Since we use complex conjugated terms, the Lagrangian (17) is real; furthermore, it is of gradation 0.

Let us now perform in (17) the following change of variables

$$
\begin{array}{llll}
\tilde{A}_{1}=\frac{\tilde{A}+\tilde{A}^{\prime}}{\sqrt{2}}, & \tilde{A}_{2}=i \frac{\tilde{A}-\tilde{A}^{\prime}}{\sqrt{2}}, & B_{1}=\frac{B+B^{\prime}}{\sqrt{2}}, & B_{2}=i \frac{B-B^{\prime}}{\sqrt{2}}, \\
\tilde{\tilde{B}}_{1}=\frac{\tilde{\tilde{B}}+\tilde{\tilde{B}}^{\prime}}{\sqrt{2}}, & \tilde{\tilde{B}}_{2}=i \frac{\tilde{\tilde{B}}-\tilde{\tilde{B}}^{\prime}}{\sqrt{2}}, & \varphi_{1}=\frac{\varphi+\varphi^{\prime}}{\sqrt{2}}, & \varphi_{2}=i \frac{\varphi-\varphi^{\prime}}{\sqrt{2}} \\
\tilde{\tilde{\varphi}}_{1}=\frac{\tilde{\tilde{\varphi}}+\tilde{\tilde{\varphi}}^{\prime}}{\sqrt{2}}, & \tilde{\tilde{\varphi}}_{2}=i \frac{\tilde{\tilde{\varphi}}-\tilde{\tilde{\varphi}}^{\prime}}{\sqrt{2}} . &
\end{array}
$$

The Lagrangian writes now

$$
\begin{aligned}
\mathcal{L}_{0}= & \partial_{m} \varphi_{1} \partial^{m} \tilde{\tilde{\varphi}}_{1}-\partial_{m} \varphi_{2} \partial^{m} \tilde{\tilde{\varphi}}_{2} \\
& +\frac{1}{6} H_{1 m n p} \tilde{\tilde{H}}_{1}^{m n p}+\partial^{n} B_{1 n m} \partial_{p} \tilde{\tilde{B}}_{1}^{p m}-\frac{1}{6} H_{2 m n p} \tilde{\tilde{H}}_{2}^{m n p}-\partial^{n} B_{1 n m} \partial_{p} \tilde{\tilde{B}}_{1}^{p m} \\
& -\frac{1}{4} F_{1 m n} F_{1}^{m n}+\frac{1}{4} \tilde{F}_{2 m n} \tilde{F}_{2}^{m n}-\frac{1}{2}\left(\partial_{m} \tilde{A}_{1}^{m}\right)^{2}+\frac{1}{2}\left(\partial_{m} \tilde{A}_{2}^{m}\right)^{2} .
\end{aligned}
$$

Notice at this point that by the redefinition (19), we find ourselves with 2 -forms $B_{1}, B_{2}, \tilde{\tilde{B}}_{1}$ and $\tilde{\tilde{B}}_{2}$ which are neither self-dual nor anti-self-dual. Moreover, one observes ${ }^{\star} B_{1}=B_{2},{ }^{\star} \tilde{\tilde{B}}_{1}=$ $\tilde{\tilde{B}}_{2}$. Therefore, one can eliminate two of them, for example $B_{2}$ and $\tilde{\tilde{B}}_{2}$; thus $\mathcal{L}_{0}$ now becomes

$$
\begin{aligned}
\mathcal{L}_{0}= & \partial_{m} \varphi_{1} \partial^{m} \tilde{\tilde{\varphi}}_{1}-\partial_{m} \varphi_{2} \partial^{m} \tilde{\tilde{\varphi}}_{2}+\frac{1}{6} H_{1 m n p} \tilde{\tilde{H}}_{1}^{m n p}+\partial^{n} B_{1 n m} \partial_{p} \tilde{\tilde{B}}_{1}^{p m} \\
& -\frac{1}{4} F_{1 m n} F_{1}^{m n}+\frac{1}{4} \tilde{F}_{2 m n} \tilde{F}_{2}^{m n}-\frac{1}{2}\left(\partial_{m} \tilde{A}_{1}^{m}\right)^{2}+\frac{1}{2}\left(\partial_{m} \tilde{A}_{2}^{m}\right)^{2} .
\end{aligned}
$$

Proceeding with the analysis of this Lagrangian, one notices that the terms in the first line of (20) are not diagonal. For this purpose, we now define

$$
\begin{array}{ll}
\hat{\varphi}_{1}=\frac{\varphi_{1}+\tilde{\tilde{\varphi}}_{1}}{\sqrt{2}}, \quad \hat{\hat{\varphi}}_{1}=\frac{\varphi_{1}-\tilde{\tilde{\varphi}}_{1}}{\sqrt{2}}, \quad \hat{\varphi}_{2}=\frac{\varphi_{2}+\tilde{\tilde{\varphi}}_{2}}{\sqrt{2}}, \quad \hat{\hat{\varphi}}_{2}=\frac{\varphi_{2}-\tilde{\tilde{\varphi}}_{2}}{\sqrt{2}}, \\
\hat{B}_{1}=\frac{B_{1}+\tilde{\tilde{B}}_{1}}{\sqrt{2}}, \quad \hat{\hat{B}}_{1}=\frac{B_{1}-\tilde{\tilde{B}}_{1}}{\sqrt{2}}
\end{array}
$$


and, with the new fields, $\mathcal{L}_{0}$ writes

$$
\begin{aligned}
\mathcal{L}_{0}= & \frac{1}{2} \partial_{m} \hat{\varphi}_{1} \partial^{m} \hat{\varphi}_{1}-\frac{1}{2} \partial_{m} \hat{\hat{\varphi}}_{1} \partial^{m} \hat{\hat{\varphi}}_{1}-\frac{1}{2} \partial_{m} \hat{\varphi}_{2} \partial^{m} \hat{\varphi}_{2}+\frac{1}{2} \partial_{m} \hat{\hat{\varphi}}_{2} \partial^{m} \hat{\hat{\varphi}}_{2} \\
& -\frac{1}{4} \tilde{F}_{1 m n} \tilde{F}_{1}^{m n}+\frac{1}{4} \tilde{F}_{2 m n} \tilde{F}_{2}^{m n}-\frac{1}{2}\left(\partial_{m} \tilde{A}_{1}^{m}\right)^{2}+\frac{1}{2}\left(\partial_{m} \tilde{A}_{2}^{m}\right)^{2} \\
& +\frac{1}{6} \hat{H}_{1 m n p} \hat{H}_{1}^{m n p}+\partial^{n} \hat{B}_{1 n m} \partial_{p} \hat{B}_{1}^{p m}-\frac{1}{6} \hat{\hat{H}}_{1 m n p} \hat{\hat{H}}_{1}^{m n p}-\partial^{n} \hat{\hat{B}}_{1 n m} \partial_{p} \hat{\hat{B}}_{1}^{m m} .
\end{aligned}
$$

The above Lagrangian writes also as

$$
\begin{aligned}
\mathcal{L}_{0}= & \frac{1}{2} \partial_{m} \hat{\varphi}_{1} \partial^{m} \hat{\varphi}_{1}-\frac{1}{2} \partial_{m} \hat{\hat{\varphi}}_{1} \partial^{m} \hat{\hat{\varphi}}_{1}-\frac{1}{2} \partial_{m} \hat{\varphi}_{2} \partial^{m} \hat{\varphi}_{2}+\frac{1}{2} \partial_{m} \hat{\hat{\varphi}}_{2} \partial^{m} \hat{\hat{\varphi}}_{2} \\
& -\frac{1}{2} \partial_{m} \tilde{A}_{1 n} \partial^{m} \tilde{A}_{1}^{n}+\frac{1}{2} \partial_{m} \tilde{A}_{2 n} \partial^{m} \tilde{A}_{2}^{n}+\frac{1}{4} \partial_{m} \hat{B}_{1 n p} \partial^{m} \hat{B}_{1}^{n p}-\frac{1}{4} \partial_{m} \hat{\hat{B}}_{1 n p} \partial^{m} \hat{\hat{B}}_{1}^{n p} .
\end{aligned}
$$

This form is actually suitable for further diagonalisation computations (see Subsection 5.3).

Let us now consider the general gauge transformations

$$
A_{m} \rightarrow A_{m}+\partial_{m} \chi, \quad B_{m n} \rightarrow B_{m n}+\partial_{m} \chi_{n}-\partial_{n} \chi_{m}
$$

where $\chi$ and $\chi_{m}$ are the gauge parameters. Hence one sees in the Lagrangian (22) presence of kinetic terms and Feynman gauge fixing terms (of type $-\frac{1}{2}\left(\partial_{m} A^{m}\right)^{2}$ for a generic vector field $A$ or of type $\partial^{n} B_{n m} \partial_{p} B^{p m}$ for a generic 2 -form $B$ ). These gauge fixing terms are not just a choice of gauge, but they are required by the invariance.

The gauge fixing terms above (of type $-\frac{1}{2}\left(\partial_{m} A^{m}\right)^{2}$ and resp. $\partial^{n} B_{n m} \partial_{p} B^{p m}$ ) imply some constraints on the gauge parameters defined in (24), namely

$$
\partial^{m} \partial_{m} \chi=0, \quad \partial^{m}\left(\partial_{m} \chi_{n}-\partial_{n} \chi_{m}\right)=0 .
$$

We will come back on this issue of gauge transformation in Subsection 5.4. The presence of these gauge fixing terms has a lot of consequences on different aspects of the model.

The first of them is related to the number of degrees of freedom of our fields. A $p$-form in $D$ dimensions has $C_{D}^{p}$ independent components. Let us now introduce for simplification differential forms notations, namely $A_{[p]}$ for a generic $p$-form, $d$ for the exterior derivative (mapping a $p$-form into a $(p+1)$-form) and for $d^{\dagger}$ for its adjoint (mapping a $p$-form into a $(p+1)$-form).

If one deals with a generic free $p$-form $\omega_{[p]}$, then the gauge transformation is

$$
\omega_{[p]} \rightarrow \omega_{[p]}+d \chi_{[p-1]},
$$

where the gauge parameter $\chi_{[p-1]}$ is a $(p-1)$-form. Using the reducibility character of the gauge transformation, one obtains at the end degrees of freedom for such a free off-shell $p$-form (see for example [32]).

Consider for example the well-known case of a photon, a 1-form, in four dimensions, we have $C_{3}^{1}=3$ degrees of freedom. To find the well-known number of 2 degrees of freedom for a physical photon, one uses Ward identities (for a detailed analysis see for example [33]).

For the general case of a on-shell $p$-form $(p \leq D-2)$ in $D$ dimensions, similar Ward identities lead to $C_{D-2}^{p}$ physical degrees of freedom.

This is not the case for the fields here; the gauge parameters are subject to constraints of type (25); thus one cannot anymore eliminate degrees of freedom of the $p$-form as was the case before. Hence, the $p$-form has $C_{D}^{p}$ degrees of freedom ( 4 for a vector field and 6 for a 2 -form).

Another important aspect of the Lagrangian (23) is that the fields

$$
\hat{\hat{\varphi}}_{1}, \quad \hat{\varphi}_{2}, \quad \tilde{A}_{2}, \quad \hat{\hat{B}}_{1}
$$


have wrong sign for their kinetic term. This implies a priori a problem of unboundedness from below of their potentials. This problem might by corrected by suitable self-interaction terms, but as we will see in the following section this is not the case because this type of terms is not allowed by this symmetry.

A possible solution to the problem of unboundness from below is based on Hodge duality of $p$-forms. Recall that for the situation of free forms, dualisation is performed at the level of the field strength; this implies equivalence of the theories of a $p$-form and a $(D-2-p)$-form (in $D$ dimensions). Indeed, starting from a generic $p$-form $\omega_{[p]}$, one considers its field strength, $d \omega_{[p]}$ which is a $(p+1)$-form. Considering its Hodge dual, we have a $(D-p-1)$-form, which is the field strength of $(D-p-2)$-form. One can see that these theories are equivalent, having the same number of physical degrees of freedom $C_{D-2}^{p}=C_{D-2}^{D-p-2}$. This can be written schematically as

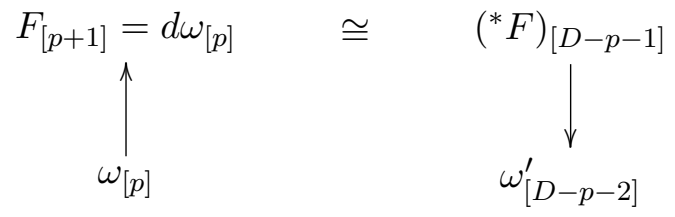

In [9], a different type of dualisation is proposed for our model. This dualisation is performed at the level of the potential directly and not at the level of its field strength, as above. This means that one replaces the fields (26) which have wrong signs in the Lagrangian, by their Hodge dual fields. Using appropriate identities relating $p$-forms kinetic terms and their Hodge duals, one has correct signs for these Hodge dual fields (see [9] for details).

Finally, notice that because of the gauge fixation present here, any $p$-form and its Hodge dual (with whom we have replaced in the Lagrangian the $p$-forms (26)) will have the same number of degrees of freedom $C_{D}^{D-p}=C_{D}^{p}$.

\subsection{Coupling between interlaced multiplets}

So far we have analysed invariant terms that arise from couplings of conjugated multiplets, $\boldsymbol{\Xi}_{++}-\boldsymbol{\Xi}_{--}$and $\boldsymbol{\Xi}_{+-}-\boldsymbol{\Xi}_{-+}$. We now look closer to couplings between the pairs of interlaced multiplets, $\boldsymbol{\Xi}_{++}-\boldsymbol{\Xi}_{+-}$and $\boldsymbol{\Xi}_{--}-\boldsymbol{\Xi}_{-+}$. We prove that quadratic coupling terms between these pairs are also invariant.

Starting the calculations with the fields given in (14), one can write the following 0-graded, real Lagrangian

$$
\begin{aligned}
\mathcal{L}_{\mathrm{c}}= & \mathcal{L}_{\mathrm{c}}\left(\boldsymbol{\Xi}_{++}, \boldsymbol{\Xi}_{+-}\right)+\mathcal{L}_{\mathrm{c}}\left(\boldsymbol{\Xi}_{--}, \boldsymbol{\Xi}_{-+}\right) \\
= & \lambda\left(\partial_{m} \varphi \tilde{\tilde{A}}^{\prime}{ }^{m}+\partial_{m} \tilde{\tilde{\varphi}} A^{\prime m}-\partial_{m} \tilde{A}^{m} \tilde{\varphi}^{\prime}-\partial_{m} \tilde{A}_{n} \tilde{B}^{\prime m n}+\partial^{m} B_{m n} \tilde{\tilde{A}}^{\prime}{ }^{n}+\partial^{m} \tilde{\tilde{B}}_{m n} A^{\prime n}\right) \\
& +\lambda^{\star}\left(\partial_{m} \varphi^{\prime} \tilde{\tilde{A}}^{m}+\partial_{m} \tilde{\tilde{\varphi}}^{\prime} A^{m}-\partial_{m} \tilde{A}^{\prime m} \tilde{\varphi}-\partial_{m} \tilde{A}_{n}^{\prime} \tilde{B}^{m n}+\partial^{m} B_{m n}^{\prime} \tilde{\tilde{A}}^{n}+\partial^{m} \tilde{\tilde{B}}_{m n}^{\prime} A^{n}\right),
\end{aligned}
$$

with $\lambda=\lambda_{1}+i \lambda_{2}$ a complex coupling constant with mass dimension.

To study the invariance of (27) one may study separately the invariance of $\mathcal{L}_{\mathrm{c}}\left(\boldsymbol{\Xi}_{++}, \boldsymbol{\Xi}_{+-}\right)$ and $\mathcal{L}_{\mathrm{c}}\left(\boldsymbol{\Xi}_{--}, \boldsymbol{\Xi}_{-+}\right)$because they do not mix under 3SUSY transformations (15). Up to total derivative, one has $\delta_{v} \mathcal{L}_{\mathrm{c}}\left(\boldsymbol{\Xi}_{++}, \boldsymbol{\Xi}_{+-}\right)=0$.

A few remarks are in order to be made now. First, one can check that the gauge fixation of $\mathcal{L}_{0}$ and $\mathcal{L}_{0}^{\prime}$ is still demanded by the terms of $\mathcal{L}_{c}$ (the last two lines of equation (29)). Indeed, if one looks at the 2 -form $B$, then terms of type $\frac{1}{2} B^{m n} F_{m n}$ fix the gauge, while terms of type ${ }^{\star} B^{m n} F_{m n}$ are gauge invariant. These terms, known as $B F$-terms are related to topological theories [34, 35, 36, 37]. Nevertheless, this line of work is not the one used here (for example we have never been concerned with surface terms in any of our invariance calculations). 
A last thing to notice is that couplings like $A^{m} \partial_{m} \varphi$ present in (29) are of Goldstone type. Usually, they are gauged away and are responsible for appearance of mass (see for example [33]). However, one sees that this mechanism cannot be applied in the case of our model, since, as we have already stated above, the gauge is partially fixed.

\subsection{Diagonalisation of the total Lagrangian}

The total Lagrangian to be considered is

$$
\mathcal{L}_{t}=\mathcal{L}_{0}+\mathcal{L}_{0}^{\prime}+\mathcal{L}_{c}
$$

where $\mathcal{L}_{0}$ and $\mathcal{L}_{0}^{\prime}$ are given in (17) and resp. (18) and $\mathcal{L}_{c}$ is given in (27). Since $\mathcal{L}$ is quadratic in the fields, we deal with a non-interacting theory and it should be possible, by field redefinitions to write the Lagrangian in a diagonal form.

In order to do this, we first perform the changes of variable (19) and (21) that make $\mathcal{L}_{0}$ explicitly real and diagonal. Obviously, the same redefinitions (keeping the same type of notations for the redefined fields) must be made for $\mathcal{L}_{0}^{\prime}$. After all this, the field content is:

6 scalar fields, $\hat{\varphi}_{1}, \hat{\hat{\varphi}}_{1}, \hat{\varphi}_{2}, \hat{\hat{\varphi}}_{2}\left(\right.$ in $\left.\mathcal{L}_{0}\right), \tilde{\varphi}_{1}, \tilde{\tilde{\varphi}}_{2}\left(\right.$ in $\left.\mathcal{L}_{0}^{\prime}\right)$;

6 vector fields, $\tilde{A}_{1}, \tilde{\tilde{A}}_{2}\left(\right.$ in $\left.\mathcal{L}_{0}\right), \hat{A}_{1}, \hat{\hat{A}}_{1}, \hat{A}_{2}, \hat{\hat{A}}_{2}\left(\right.$ in $\left.\mathcal{L}_{0}^{\prime}\right)$;

3 two-forms $\hat{B}_{1}, \hat{\hat{B}}_{1}$ (in $\left.\mathcal{L}_{0}\right) \tilde{B}_{2}\left(\right.$ in $\left.\mathcal{L}_{0}^{\prime}\right)$.

We thus have a total of 15 independent fields. Expressed with these new fields, $\mathcal{L}_{t}$ decouples into 3 distinct pieces, each of them having the exact same dependence on a set of 5 fields (two scalars, two vectors and one 2 -forms, denoted generically by $\varphi_{1}, \varphi_{2}, A_{1}, A_{2}$ and $B$ ). This Lagrangian writes

$$
\begin{aligned}
\mathcal{L}\left(\varphi_{1}, \varphi_{2}, A_{1}, A_{2}, B\right)= & \frac{1}{2}\left(\partial_{m} \varphi_{1}\right)^{2}-\frac{1}{2}\left(\partial_{m} \varphi_{2}\right)^{2}-\frac{1}{2}\left(\partial_{m} A_{1 n}\right)^{2}+\frac{1}{2}\left(\partial_{m} A_{2 n}\right)^{2}+\frac{1}{4}\left(\partial_{m} B_{n p}\right)^{2} \\
& +\lambda_{1}\left(A_{1}{ }^{m} \partial_{m} \varphi_{1}+A_{2}{ }^{m} \partial_{m} \varphi_{2}-B^{m n} \partial_{m} A_{1 n}-{ }^{\star} B^{m n} \partial_{m} A_{2 n}\right) \\
& +\lambda_{2}\left(-A_{2}{ }^{m} \partial_{m} \varphi_{1}+A_{1}{ }^{m} \partial_{m} \varphi_{2}+B^{m n} \partial_{m} A_{2 n}-{ }^{\star} B^{m n} \partial_{m} A_{1 n}\right) .(29)
\end{aligned}
$$

Thus, for diagonalising the total Lagrangian $\mathcal{L}_{t}$ it is enough to work on $\mathcal{L}\left(\varphi_{1}, \varphi_{2}, A_{1}, A_{2}, B\right)$. Expressing the Lagrangian in the Fourier space, the first step is to complete a perfect square for the terms involving $\tilde{A}_{1}$ (we denote in the rest of this subsection the Fourier transforms by tilde, not to be confused with the tilde in the fields we had until equation (29)). One has to define

$$
\tilde{A}_{1 m}^{\prime}(p)=\tilde{A}_{1 m}(p)+\frac{\lambda_{1}}{p^{2}} i p_{m} \tilde{\varphi}_{1}(p)+\frac{\lambda_{2}}{p^{2}} i p_{m} \tilde{\varphi}_{2}(p)+\frac{\lambda_{1}}{p^{2}} i p^{r} \tilde{B}_{r m}(p)+\frac{\lambda_{2}}{p^{2}} i p^{r}\left({ }^{\star} \tilde{B}_{r m}(p)\right) .
$$

The next step is to complete a perfect square for the terms involving $\tilde{A}_{2}$ (the Fourier transform of the vector field $A_{1}$ ); one defines

$$
\tilde{A}_{2 m}^{\prime}(p)=\tilde{A}_{2 m}(p)-\frac{\lambda_{1}}{p^{2}} i p_{m} \tilde{\varphi}_{2}(p)+\frac{\lambda_{2}}{p^{2}} i p_{m} \tilde{\varphi}_{1}(p)+\frac{\lambda_{2}}{p^{2}} i p^{r} \tilde{B}_{r m}(p)-\frac{\lambda_{1}}{p^{2}} i p^{r}\left({ }^{\star} \tilde{B}_{r m}(p)\right) .
$$

A final diagonalisation can be written for $\tilde{\varphi}$. Thus one defines

$$
\tilde{\varphi}^{\prime}(p)=\tilde{\varphi}(p)+\frac{\lambda_{1} \lambda_{2}}{\frac{1}{2}\left(p^{2}-\left(\lambda_{2}^{2}-\lambda_{1}^{2}\right)\right)} \tilde{\varphi}_{2}(p) .
$$

After all this, the Lagrangian finally writes as

$$
\tilde{\mathcal{L}}=\frac{1}{2}\left(p^{2}-\left(\lambda_{2}^{2}-\lambda_{1}^{2}\right)\right) \tilde{\varphi}_{1}^{\prime}(p) \tilde{\varphi}_{1}^{\prime}(-p)
$$




$$
\begin{aligned}
& -\frac{1}{2}\left(p^{2}-\left(\lambda_{2}^{2}-\lambda_{1}^{2}\right)+\frac{\lambda_{1}^{2} \lambda_{2}^{2}}{\frac{1}{2}\left(p^{2}-\left(\lambda_{2}^{2}-\lambda_{1}^{2}\right)\right)}\right) \tilde{\varphi}_{2}(p) \tilde{\varphi}_{2}(-p) \\
& -\frac{1}{2} p^{2} \tilde{A}_{1 m}^{\prime}(p) \tilde{A}_{1}^{\prime m}(-p)+\frac{1}{2} p^{2} \tilde{A}_{2 m}^{\prime}(p) \tilde{A}_{2}^{\prime m}(-p)+\frac{1}{4} p^{2} \tilde{B}_{m n}(p) \tilde{B}^{m n}(-p) \\
& +\frac{1}{2} \frac{1}{p^{2}} p^{r} p_{s}\left(\lambda_{1}^{2}-\lambda_{2}^{2}\right)\left(\tilde{B}_{r m}(p) \tilde{B}^{s m}(-p)-{ }^{\star} \tilde{B}_{r m}(p)^{\star} \tilde{B}^{s m}(-p)\right) \\
& +\frac{\lambda_{1} \lambda_{2}}{p^{2}} p^{r} p_{s}\left(\tilde{B}_{r m}(p)^{\star} \tilde{B}^{s m}(-p)+\tilde{B}^{s m}(p)^{\star} \tilde{B}_{r m}(-p)\right) .
\end{aligned}
$$

One can now remark that not all values of the parameters $\lambda_{1}$ and $\lambda_{2}$ are allowed if we do not want tachyons to be present. Some allowed values (which considerably simplify the Lagrangian (30)) are $\lambda_{1}=\lambda_{2}$ or $\lambda_{1}=0$. However one remarks a non-conventional form of the kinetic term for the 2 -form $B$. One final remark is that we have done this diagonalisation on the Lagrangian without dualisation mentioned at the end of Subsection 5.1; the sign of the kinetic terms will not change; however the same type of calculation may be performed for the dualised Lagrangian.

One more remark is to be made. Even if we have so far considered non-massive fields, invariant mass terms can be explicitly added to our Lagrangian (see [9]). Moreover, as one can easily see from the 3SUSY algebra (1), $P^{2}$ is a Casimir operator and therefore all states in an irreducible representation must have the same mass.

The analysis done so far for the massless multiplets do not drastically change. For example, in the massive case one has no gauge invariance and the number of degrees of freedom remains the same (one does not have gauge parameters to eliminate any degree of freedom). For diagonalisation of the total free Lagrangian, these mass terms do not change our analysis qualitatively.

\subsection{Compatibility with Abelian gauge invariance}

We now look closer to the problem of the compatibility with Abelian gauge symmetry. We have seen that these two symmetries are closely connected, in the sense that the gauge symmetry is fixed by the Feynman gauge fixing terms required in the Lagrangian (see Subsections 5.1 and 5.3). So far we have also seen some of the consequences of this gauge fixing, such as possibility of dualisation related to the equal number of degrees of freedom (see Subsection 5.1) and also impossibility of gauging away the coupling terms of $\mathcal{L}_{c}(27)$.

Nevertheless, another question is appropriate at this level. If one acts with the gauge transformation on a multiplet will the result be a multiplet? Or, schematically, $\boldsymbol{\Xi} \stackrel{\text { gauge }}{\longrightarrow} \boldsymbol{\Xi}^{\prime}$ ? So what one has to check is whether or not $\boldsymbol{\Xi}^{\prime}$ is a 3SUSY multiplet. (Recall that this is the case for SUSY, where a gauge transformation transforms a vector superfield $V$ to $V+\Phi+\Phi^{\dagger}$, with $\Phi$ a chiral superfield (see for example [25]).) Moreover we also find in what conditions gauge parameters may form a 3SUSY multiplet.

I. Let us first write the general gauge transformation one uses for the physical fields $\hat{\varphi}_{1}, \hat{\varphi}_{2}$, $\hat{\hat{\varphi}}_{1}, \hat{\hat{\varphi}}_{2}, \tilde{A}_{1}, \tilde{A}_{2}, \hat{B}_{1}, \hat{\hat{B}}_{1}$

$$
\hat{\varphi}_{1} \rightarrow \hat{\varphi}_{1}+\hat{k}_{1}, \quad \tilde{A}_{1 m} \rightarrow \tilde{A}_{1 m}+\partial_{m} \tilde{\chi}_{1}, \quad \hat{B}_{1 m n} \rightarrow \hat{B}_{1 m n}+\partial_{m} \hat{\chi}_{1 n}-\partial_{n} \hat{\chi}_{1 m} .
$$

and similarly for the rest of the fields ( $\hat{k}_{1}$ being some constant). Recall that these physical fields were obtained from linear redefinitions of the original fields (14). Hence one can write down gauge transformations of the fields (14) also. For example, the vector field $\tilde{A}_{1}$ was obtained from the fields $\tilde{A}$ and $\tilde{A}^{\prime}$ by the redefinition $(19), \tilde{A}_{1}=\frac{1}{\sqrt{2}}\left(\tilde{A}+\tilde{A}^{\prime}\right)$. Hence the gauge parameter $\tilde{\chi}_{1}$ of $(31)$ is written as $\tilde{\chi}_{1}=\frac{1}{\sqrt{2}}\left(\tilde{\chi}+\tilde{\chi}^{\prime}\right)$ that will thus allow us to obtain the gauge parameters $\tilde{\chi}$ and $\tilde{\chi}^{\prime}$ of the field $\tilde{A}$ and respectively $\tilde{A}^{\prime}$. 
The gauge transformations for the original fields (14) thus write

$$
\phi \rightarrow \phi+k, \quad \tilde{A}_{m} \rightarrow \tilde{A}_{m}+\partial_{m} \tilde{\chi}, \quad B_{m n}^{( \pm)} \rightarrow B_{m n}^{( \pm)}+\partial_{m} \chi_{n}-\partial_{n} \chi_{m} \mp i \varepsilon_{m n p q} \partial^{p} \chi^{q}
$$

and similarly for the rest of the fields. Note, however, that for the case of 2-forms, compatibility between the transformations (31) and (32) needs a closer look. Indeed, write in $p$-form notation the transformation (32) for the self-dual 2-form $B_{m n}$

$$
B \rightarrow B+d \chi_{[1]}-i^{*}\left(d \chi_{[1]}\right)
$$

Recall now that $B^{\prime}=B^{*}$; one has

$$
B^{\prime} \rightarrow B^{\prime}+d \chi_{[1]}^{*}+i^{*}\left(d \chi_{[1]}^{*}\right) .
$$

(One should pay attention to the notations used for dual and complex conjugation, that is ${ }^{*} B$ denotes the dual of $B$ whereas $B^{*}$ denotes the complex conjugated of $B$.) Equation (19) combined these two 2 -forms in the real 2-form $B_{1}=\frac{1}{\sqrt{2}}\left(B+B^{\prime}\right)$. Thus, its gauge transformation writes

$$
B_{1} \rightarrow B_{1}+\frac{1}{\sqrt{2}}\left(d\left(\chi_{[1]}+\chi_{[1]}^{*}\right)-i^{*} d\left(\chi_{[1]}-\chi_{[1]}^{*}\right)\right) .
$$

One immediate solution for (35) to be the gauge transformation (31) for a 2-form, is to impose that the 1 -form $\chi_{[1]}$ is real.

However, we now prove that this compatibility can still be achieved even if the 1 -form $\chi_{[1]}$ is complex. For this denote by $\lambda_{[1]}=\frac{1}{\sqrt{2}}\left(\chi_{[1]}+\chi_{[1]}^{*}\right)$ and $\lambda_{[3]}=-i \frac{1}{\sqrt{2}} *\left(\chi_{[1]}-\chi_{[1]}^{*}\right)$. Notice that $\lambda_{[1]}$ and $\lambda_{[3]}$ are real. One has ${ }^{* *}\left(\chi_{[1]}-\chi_{[1]}^{*}\right)=\chi_{[1]}-\chi_{[1]}^{*}$ and using also the definition $d^{\dagger}={ }^{*} d^{*}$, equation (35) writes

$$
B_{1} \rightarrow B_{1}+d \lambda_{[1]}+d^{\dagger} \lambda_{[3]} .
$$

We now prove that for a 2 -form $B_{1}$ one can write a gauge transformation as $B_{1} \rightarrow B_{1}+d \lambda_{[1]}$, with $d^{\dagger} d \lambda_{[1]}=0$ but also as $B_{1} \rightarrow B_{1}+d^{\dagger} \lambda_{[3]}$ with $d d^{\dagger} \lambda_{[3]}=0$. Indeed, since $d^{\dagger} d \lambda_{[1]}=0$ this means that there exists a 3 -form $\lambda_{[3]}$ such that $d \lambda_{[1]}=d^{\dagger} \lambda_{[3]}$. Thus, the gauge transformation can be written as $B_{1} \rightarrow B_{1}+d^{\dagger} \lambda_{[3]}$. Moreover, adding this two equivalent types of gauge transformations one can write a "general" gauge transformation for $B_{1}$ as in (36). This means that (31) and (32) are compatible for the 2 -forms even if the gauge parameter $\chi_{[1]}$ is complex.

We now show what are the constraints on the gauge parameters $k, \tilde{\chi}$ and $\chi_{m}$ of the the gauge transformations (32). As before, the case of the scalar and vector gauge parameter is simple, leading to

$$
\partial_{m} k=0 \quad \text { and } \quad \square \tilde{\chi}=0 .
$$

For the case of a 2-form $B^{( \pm)}$, one checks separately the invariance under $(33)$ of $\left(d B^{( \pm)}\right)^{2}$ and $\left(d^{\dagger} B^{( \pm)}\right)^{2}{ }^{3}$. This gives $d^{\dagger} d \chi_{[1]}=0$. Hence, these constraints on a general gauge parameter $\chi_{[p]}$ write

$$
d^{\dagger} d \chi_{[p]}=0 .
$$

In our particular case, using component notations, one has

$$
\partial_{m} k=0, \quad \square \tilde{\chi}=0, \quad \square \chi_{n}-\partial_{n} \partial_{m} \chi^{m}=0 .
$$

\footnotetext{
${ }^{3}$ Note that for the case of a real 2-form the gauge parameter $\chi_{[1]}^{\prime}$ defined in (31) is from the same reason subject to the constraint $d^{\dagger} d \hat{\chi}_{[1]}=0$.
} 
The strategy we adopt here is to find explicit forms of the gauge parameters defined in (32) and subject to the constraints (38).

II. The second step of our analysis is to have some transformations of a multiplet into a multiplet of same type, transformation that can then be matched with the gauge transformation (32). Since in Subsection 4.4 we have introduced the derivative multiplets (that transform like 3SUSY multiplets), we can use them to do the job:

$$
\boldsymbol{\Xi}_{++} \rightarrow \boldsymbol{\Xi}_{++}+\mathcal{D} \boldsymbol{\Xi}_{+-}
$$

For instance, one can use the derivative of a multiplet $\boldsymbol{\Xi}_{+-}=\left(\lambda_{m}, \tilde{\lambda}, \tilde{\lambda}_{m n}, \tilde{\tilde{\lambda}}_{m}\right)$, writing thus (39) as

$$
\begin{aligned}
& \varphi \rightarrow \varphi+\partial_{m} \lambda^{m}, \quad B_{m n} \rightarrow B_{m n}+\partial_{m} \lambda_{n}-\partial_{n} \lambda_{m}-i \varepsilon_{m n p q} \partial^{p} \lambda^{q}, \\
& \tilde{A}_{m} \rightarrow \tilde{A}_{m}+\partial_{m} \tilde{\lambda}+\partial^{n} \tilde{\lambda}_{n m}, \quad \tilde{\tilde{\varphi}} \rightarrow \tilde{\tilde{\varphi}}+\partial^{m} \tilde{\tilde{\lambda}}_{m}, \\
& \tilde{\tilde{B}}_{m n} \rightarrow \tilde{\tilde{B}}_{m n}+\partial_{m} \tilde{\tilde{\lambda}}_{n}-\partial_{n} \tilde{\tilde{\lambda}}_{m}-i \varepsilon_{m n p q} \partial^{p} \tilde{\tilde{\lambda}}^{q} .
\end{aligned}
$$

III. The last step of this programme is to make (40) a gauge transformation, that is to match it with (32) and the conditions $(38)^{4}$.

First remark that the actual matching of these transformations implies a non-trivial condition for the parameters of the transformation of the vector field, namely

$$
\partial^{n} \tilde{\lambda}_{n m}=\partial_{m} \chi
$$

and, since $\tilde{\lambda}_{n m}$ is antisymmetric, one has

$$
\square \chi=0 .
$$

Now, imposing the conditions (38) on the set of gauge parameters $\left(\lambda_{m} ; \tilde{\lambda}_{,} \tilde{\lambda}_{m n} ; \tilde{\tilde{\lambda}}_{m}\right)$, one has

$$
\begin{aligned}
& \partial_{m}(\partial \cdot \lambda)=\partial_{m}(\partial \cdot \tilde{\tilde{\lambda}})=0, \\
& \square \lambda_{m}=\square \tilde{\tilde{\lambda}}_{m}=0, \\
& \square \tilde{\lambda}+\partial^{m} \partial^{n} \tilde{\lambda}_{n m} \equiv \square \tilde{\lambda}=0 .
\end{aligned}
$$

We have now to find explicit solutions of the gauge parameters which are compatible with all these constraints. In order to do this,

1) we determine the solutions for $\tilde{\lambda}, \chi$ satisfying the constraints (45), (42);

2) knowing $\chi$, we then construct an anti-self-dual 2-form $\tilde{\lambda}_{m n}$ satisfying (41);

3) we finally find explicit solutions for $\lambda_{m}, \tilde{\tilde{\lambda}}_{m}$ satisfying (43), (44).

Existence of these solutions would prove at this step compatibility between 3SUSY and the Abelian gauge invariance.

1. If the scalar functions $\tilde{\lambda}$ and $\chi$ depend only on the space-time Lorentz invariant $x_{m} x^{m}$, then the conditions (45), (42) determine uniquely their form, $\tilde{\lambda}\left(x^{2}\right) \propto \chi\left(x^{2}\right) \propto 1 / x^{2}$ up to some additive constants. In the context of the symmetry (1), whose generators and transformation parameters are 4-vectors, it is somewhat natural to include dependence of a 4 -vector $\xi_{m}$. Moreover, by analysing solutions of (45) and (42) when $\xi^{2}$ is equal to or different from 0 , we find

\footnotetext{
${ }^{4}$ Since these equations have practically the same form, it now becomes clear why we have chosen to work with gauge transformations of type (32) and not the gauge transformations (31) of the real fields since we work directly on the $\boldsymbol{\Xi}_{++}$.
} 
more general configurations for $\lambda$ and $\chi$ when $\xi^{2}=0$ (see [9]). Hence we explicitly treat this case in this subsection.

We thus have to treat an equation of type

$$
\square f\left(x^{2}, x \cdot \xi\right)=0,
$$

where $f$ denotes generically $\tilde{\lambda}$ or $\chi$. A solution for this equation is given by

$$
f\left(x^{2}, \xi \cdot x\right)=G(\xi \cdot x)+(\xi \cdot x)^{-1} H\left(\frac{x^{2}}{(\xi \cdot x)}\right),
$$

where $\mathrm{G}$ and $\mathrm{H}$ are arbitrary functions.

This provides us an explicit form for the parameters $\tilde{\lambda}$ or $\chi$

$$
\begin{aligned}
& \tilde{\lambda}\left(\xi \cdot x, x^{2}\right)=G_{1}(\xi \cdot x)+(\xi \cdot x)^{-1} H_{1}\left(\frac{x^{2}}{(\xi \cdot x)}\right), \\
& \chi\left(\xi \cdot x, x^{2}\right)=G_{2}(\xi \cdot x)+(\xi \cdot x)^{-1} H_{3}\left(\frac{x^{2}}{(\xi \cdot x)}\right),
\end{aligned}
$$

completing thus step 1 of the programme.

2. As already stated, we now have to find a form of $\tilde{\lambda}_{m n}$ which satisfies the constraint (41), with $\chi$ given by (49) above. A possible solution is

$$
\tilde{\lambda}_{m n}\left(\xi \cdot x, x^{2}\right)=x_{[m} \xi_{n]_{-}} F\left(\xi \cdot x, x^{2}\right),
$$

where the function $F$ is expressed in terms of $G_{2}, H_{3}$ appearing in (49):

$$
F\left(\xi \cdot x, x^{2}\right)=-(\xi \cdot x)^{-2} H_{3}\left(\frac{x^{2}}{(\xi \cdot x)}\right)+(\xi \cdot x)^{-1} G_{2}(\xi \cdot x)-2(\xi \cdot x)^{-3} \int_{0}^{\xi \cdot x} G_{2}(t) t d t .
$$

3. Similarly to step 1 , we now investigate possible solutions for the gauge parameters $\lambda_{m}, \tilde{\tilde{\lambda}}_{m}$, which satisfies equations (43) and (44). We consider them as functions of the vectors $x$ and $\xi$ and, as before, we assume $\xi^{2}=0$. Hence, the problem is reduced to finding explicit solutions for

$$
\partial_{m} \partial_{p} \mathcal{A}^{p}(x, \xi)=0, \quad \square \mathcal{A}_{m}(x, \xi)=0
$$

(which are just equations (43) and (44), $\mathcal{A}$ standing for $\lambda_{m}$ or $\tilde{\tilde{\lambda}}_{m}$ ). Some solution of these equation is given by

$$
\mathcal{A}_{m}(x, \xi)=g(\xi \cdot x) \xi_{m}+\alpha x_{m}+\left(\frac{1}{\left(x^{2}\right)^{2}} \alpha_{m r}+\beta_{m r}\right) x^{r}+\kappa\left(\frac{x^{2}}{(\xi \cdot x)^{3}} \xi_{m}-\frac{x_{m}}{(\xi \cdot x)^{2}}\right),
$$

where $g$ is an arbitrary function, $\kappa, \alpha, \beta_{m n}$ arbitrary constants and $\alpha_{m n}$ an arbitrary antisymmetric tensor.

Thus one can now write the following expressions for the last gauge parameters $\lambda_{m}$ or $\tilde{\tilde{\lambda}}_{m}$

$$
\begin{aligned}
\lambda_{m}\left(\xi \cdot x, x^{2}\right)= & g_{1}(\xi \cdot x) \xi_{m}+\alpha x_{m} \\
& +\left(\frac{1}{\left(x^{2}\right)^{2}} \alpha_{m r}+\beta_{m r}\right) x^{r}+\kappa_{1}\left(\frac{x^{2}}{(\xi \cdot x)^{3}} \xi_{m}-\frac{x_{m}}{(\xi \cdot x)^{2}}\right), \\
\tilde{\tilde{\lambda}}_{m}\left(\xi \cdot x, x^{2}\right)= & \tilde{\tilde{g}}_{1}(\xi \cdot x) \xi_{m}+\tilde{\tilde{\alpha}} x_{m} \\
& +\left(\frac{1}{\left(x^{2}\right)^{2}} \tilde{\tilde{\alpha}}_{m r}+\tilde{\tilde{\beta}}_{m r}\right) x^{r}+\tilde{\tilde{\kappa}}_{1}\left(\frac{x^{2}}{(\xi \cdot x)^{3}} \xi_{m}-\frac{x_{m}}{(\xi \cdot x)^{2}}\right) .
\end{aligned}
$$


We have therefore obtained a proof of existence of gauge transformations which are compatible with our symmetry. Nevertheless, we have found constrained forms of our gauge parameters $\lambda_{m}$, $\tilde{\lambda}, \tilde{\lambda}_{m n}$ and resp. $\tilde{\tilde{\lambda}}_{m}$ (equations (54), (48), (50) and resp. (55)).

Before ending this section, let us mention that a discussion related to Noether currents was initiated in [8], mostly in relation with to the case of algebra of conserved charges compared to the case where symmetries are grouped within Lie (super)algebras. Furthermore some conserved currents were explicitly calculated in [38].

\section{$6 \quad$ Study of interaction possibilities}

In the previous section we have considered non-interacting terms allowed by 3SUSY invariance. Here we investigate the possibility of interacting terms, terms which must have a degree in the fields higher than 2 (thus not being possible to diagonalise them back to kinetic terms). The main result is that for the bosonic multiplets considered, no such interaction terms are allowed.

To approach this issue we make a systematic study of all interaction possibilities for our multiplets. We first find what are the fields $\Psi$ (content and transformation laws) that can couple to multiplets in an invariant quadratic way. We then express these fields $\Psi$ as a function $\Psi\left(\boldsymbol{\Xi}_{++}, \boldsymbol{\Xi}_{--}, \boldsymbol{\Xi}_{+-}, \boldsymbol{\Xi}_{-+}\right)$. We find this function to be linear in the multiplets; hence the most general invariant terms which can be constructed are quadratic and thus non-interacting.

\subsection{Possible couplings of a given multiplet}

We focus on the coupling of a $\boldsymbol{\Xi}_{++}$multiplet, the other cases being similar. Considering its field content, the most general possibility of quadratic coupling with some set of unknown fields $\Psi$ is

$$
\mathcal{L}\left(\boldsymbol{\Xi}_{++}, \Psi\right)=\varphi \tilde{\tilde{\psi}}+\tilde{\tilde{\varphi}} \psi+\frac{1}{4} B^{(+) m n} \tilde{\tilde{\psi}}_{m n}+\frac{1}{4} \tilde{\tilde{B}}_{m n}^{(+)} \psi^{m n}-\tilde{A}_{m} \tilde{\psi}^{m}
$$

with $\psi, \tilde{\tilde{\psi}}$ two scalars, $\tilde{\psi}_{m}$ a vector and $\psi_{m n}, \tilde{\tilde{\psi}}_{m n}$ two 2-forms which are self-dual. A priori some of the fields $\Psi$ can be set to zero.

To find the set of fields $\Psi$ we impose that (56) transforms as a total derivative. We first treat the case where the fields $\Psi$ contain no derivative terms. Applying (15) directly, one has the variation of (56) and is able to prove (see [9]):

I: If the $\psi$ fields contain no derivative terms and (56) is invariant, then they form a multiplet of type $\boldsymbol{\Xi}_{++}$.

Similar type of arguments lead to the same conclusion if the $\psi$ fields contain at most one derivative term.

Let us now allow a higher number of derivatives in the fields $\Psi$. For example, if one considers two derivatives, one can write fields of type

$$
\psi=\square \lambda, \psi_{m}=\alpha \square \lambda_{m}+\beta \partial_{m} \partial_{n} \lambda^{n}, \quad \psi_{m n}=\alpha^{\prime} \square \lambda_{m n}+\beta^{\prime} \partial^{p} \partial_{[m} \lambda_{n]+p} .
$$

Generally speaking, if one consider an even number $n$ of partial derivatives, the terms that can be added are

$$
\psi=\square \square^{\frac{n}{2}} \lambda, \quad \psi_{m}=\alpha \square^{\frac{n}{2}} \lambda_{m}+\beta \square^{\frac{n-2}{2}} \partial^{n} \partial_{m} \lambda_{n}, \quad \psi_{m r}=\alpha \square^{\frac{n}{2}} \lambda_{m r}+\beta \square^{\frac{n-2}{2}} \partial^{p} \partial_{[m} \lambda_{n]+p} .
$$

If $n$ is an odd number, one can construct

$$
\psi=\square^{\frac{n-1}{2}} \partial^{m} \lambda_{m}, \quad \psi_{m}=\alpha \square^{\frac{n-1}{2}} \partial_{m} \lambda+\beta \square^{\frac{n-1}{2}} \partial^{p} \lambda_{p m}, \quad \psi_{m p}=\square^{\frac{n-1}{2}} \partial_{[m} \lambda_{n]+} .
$$


Similar arguments lead to the same type of result $\mathbf{I}$.

We have thus seen which are the most general couplings of a given multiplet. We now have the set of fields $\Psi$, its content and transformation laws in terms of the $\lambda$ fields. In the remainder of this section we see what is the most general way one can construct these $\Psi$ fields out of the original $\boldsymbol{\Xi}_{ \pm \pm}$multiplets.

\subsection{Generalised tensor calculus}

Here also we focus on the $\boldsymbol{\Xi}_{++}$multiplet, the other cases being similar. So, what we look for is to express the fields $\psi, \psi_{m n}, \tilde{\psi}_{m}, \tilde{\tilde{\psi}}, \tilde{\psi}_{m n}$ of the previous subsection as functions of the fields of the multiplets $\boldsymbol{\Xi}_{++}, \boldsymbol{\Xi}_{--}, \boldsymbol{\Xi}_{+-}, \boldsymbol{\Xi}_{-+}$and of their derivatives.

After direct explicit calculations (see [23] for details) one proves

II. The only function $\Psi$ with at most first order derivatives in the fields and transforming as $a \boldsymbol{\Xi}_{++}$multiplet is

$$
\Psi\left(\boldsymbol{\Xi}_{++}, \boldsymbol{\Xi}_{+-}, \boldsymbol{\Xi}_{+-}, \boldsymbol{\Xi}_{--}\right)=\alpha \boldsymbol{\Xi}_{++}+\beta \boldsymbol{\Xi}_{--}^{*}+\gamma \mathcal{D} \boldsymbol{\Xi}_{+-}+\mu \mathcal{D} \boldsymbol{\Xi}_{-+}^{*}
$$

Moreover, if one considers several copies of the same multiplet, the conclusion does not change. The case of functions involving higher number of derivatives does not change the final conclusion either: the $\psi$ fields can be obtained only linearly out of the four considered bosonic multiplets. Comparing this result with the result of the previous subsection (which was stating that these $\psi$ fields are the most general possibility to quadratically couple the multiplets to some arbitrary fields) one concludes that no invariant terms of order higher than two in the fields can be constructed. This means that one cannot obtain invariant self-interacting terms for the bosonic multiplets $\boldsymbol{\Xi}_{ \pm \pm}$.

\section{Concluding remarks}

Even if we have proven in the previous section impossibility of writing invariant interaction terms, this situation can be compared with incompatibility for the usual electromagnetism, where photons do not self-interact. Hence, one may think of a possibility of constructing nonAbelian models.

More general possibilities of interaction have to be investigated for a verdict on this issue. One might reconsider at this level the fermionic multiplets of [8] and investigate a possible interaction between them and boson multiplets. Furthermore, interactions with a different type of bosonic multiplets (eventually more general multiplets) may be taken into consideration. Let us also mention that considering $p$-forms with $p \geq 2$ implies a high rigidity for the interaction possibilities, see [40]).

Some deeper analysis of possible mechanisms of elimination of unphysical degrees of freedom of the fields may be appropriate. This may eventually involve some presence of ghosts, in connection with suited quantification procedures.

In [39], G. Moultaka et. al. consider the algebra (1) in arbitrary dimensions; this leads to the implementation of a new, cubic symmetry at the level of $p$-forms. Recalling that the $p$-forms of the bosonic multiplets couple naturally to extended objects of dimension $(p-1)((p-1)$-branes); one could seek for such an invariant theory for interacting $p$-branes.

A different type of remark is to be made when one considers different types of algebraic structure mentioned in Section 1: possible useful connections in between do not seem very likely, since these algebraic structures are different from the very definition.

However, recalling the construction of [29] of a relativistic wave equation involving the cubic root of the Klein-Gordon operator, let us mention that as Dirac equation is related to SUSY, 
this cubic root of the Klein-Gordon operator is related to fractional supersymmetry. As already mentioned here in Subsection 3.3, in [16] for 1-dimensional fractional supersymmetry, the theory is localised leading to a local fractional supergravity. In the case of the $(3+1)$-dimensional model reviewed here, a generalisation in such a direction constitutes a promising perspective of future work.

Finally, let us argue on the fact that maybe before starting more field theoretical developments of this type of models, it would be more important to have a closer look at their very foundations, i.e. the implications they would have when compared to some of basic notions of field theory, like for example the assumptions of analicity of no-go theorems, canonical quantisation rules etc. This type of study may give stronger information of what may or may not be pertinent for coherent physical approaches.

\section{A Determination of the matrices $\sigma_{m n r}$}

In this appendix we show how one obtains the connection (7) between the generators $V$ and the SUSY supercharges $Q$; we thus obtain the explicit form of the matrices $\sigma_{m n r}$ appearing in equation (7).

First recall equations (5) and (6) of the algebra (1) resp. SUSY algebra:

$$
\left\{V_{m}, V_{n}, V_{r}\right\}=\eta_{m n} P_{r}+\eta_{m r} P_{n}+\eta_{r n} P_{m}, \quad\left\{Q_{\alpha}, \bar{Q}_{\dot{\beta}}\right\}=2\left(\sigma^{m}\right)_{\alpha \dot{\beta}} P_{m}
$$

(with $\alpha, \dot{\beta}=1,2$, see [25] for conventions on the SUSY algebra).

With use of the form of the Pauli matrices $\sigma^{m}$, the second equation of (57) writes explicitly

$$
\begin{aligned}
& \left\{Q_{1}, \bar{Q}_{\dot{1}}\right\}=2\left(P_{0}+P_{3}\right), \quad\left\{Q_{1}, \bar{Q}_{\dot{2}}\right\}=2\left(P_{1}-i P_{2}\right), \\
& \left\{Q_{2}, \bar{Q}_{\dot{1}}\right\}=2\left(P_{1}+i P_{2}\right), \quad\left\{Q_{2}, \bar{Q}_{\dot{2}}\right\}=2\left(P_{0}-P_{3}\right) .
\end{aligned}
$$

These equations allow one to express the momentums $P_{m}$ as a function of the SUSY algebra anticommutators:

$$
\begin{array}{ll}
P_{0}=\frac{1}{4}\left(\left\{Q_{1}, \bar{Q}_{\dot{1}}\right\}+\left\{Q_{2}, \bar{Q}_{\dot{1}}\right\}\right), & P_{1}=\frac{1}{4}\left(\left\{Q_{1}, \bar{Q}_{\dot{2}}\right\}+\left\{Q_{2}, \bar{Q}_{\dot{j}}\right\}\right), \\
P_{2}=\frac{i}{4}\left(\left\{Q_{1}, \bar{Q}_{\dot{2}}\right\}-\left\{Q_{2}, \bar{Q}_{\dot{1}}\right\}\right), & P_{3}=\frac{1}{4}\left(\left\{Q_{1}, \bar{Q}_{\dot{1}}\right\}-\left\{Q_{2}, \bar{Q}_{\dot{1}}\right\}\right) .
\end{array}
$$

One can now insert equations (59) in the first equation of (57). For example, one has

$$
\left\{V_{0}, V_{0}, V_{0}\right\}=3 P_{0}
$$

and, inserting the first of equations (59) one has

$$
\left(\sigma_{000}\right)^{1, \dot{1}}=\frac{3}{4},\left(\sigma_{000}\right)^{2, \dot{2}}=\frac{3}{4} .
$$

Similarly one gets the rest of the entries of the matrices $\sigma_{m n r}$. Thus the non-zero entries of these matrices are:

$$
\begin{array}{rlrl}
\left(\sigma_{000}\right)^{1, \dot{1}} & =\frac{3}{4}, & \left(\sigma_{000}\right)^{2, \dot{2}}=\frac{3}{4}, \\
\left(\sigma_{001}\right)^{1, \dot{2}}=\frac{1}{4}, & \left(\sigma_{001}\right)^{2, \dot{1}}=-\frac{1}{4}, \\
\left(\sigma_{002}\right)^{1, \dot{2}}=\frac{i}{4}, & \left(\sigma_{001}\right)^{2, \dot{1}}=-\frac{i}{4}, \\
\left(\sigma_{003}\right)^{1, \dot{1}}=\frac{1}{4}, & \left(\sigma_{003}\right)^{2, \dot{2}}=-\frac{1}{4},
\end{array}
$$




$$
\begin{array}{ll}
\left(\sigma_{111}\right)^{1, \dot{2}}=-\frac{3}{4}, & \left(\sigma_{111}\right)^{2, \dot{1}}=-\frac{3}{4}, \\
\left(\sigma_{112}\right)^{1, \dot{2}}=-\frac{i}{4}, & \left(\sigma_{112}\right)^{2, \dot{1}}=\frac{i}{4}, \\
\left(\sigma_{113}\right)^{1, \dot{1}}=-\frac{1}{4}, & \left(\sigma_{003}\right)^{2, \dot{2}}=\frac{1}{4}, \\
\left(\sigma_{222}\right)^{1, \dot{2}}=-\frac{3 i}{4}, & \left(\sigma_{222}\right)^{2, \dot{1}}=\frac{3 i}{4}, \\
\left(\sigma_{223}\right)^{1, \dot{1}}=-\frac{1}{4}, & \left(\sigma_{223}\right)^{2, \dot{2}}=\frac{1}{4}, \\
\left(\sigma_{333}\right)^{1, \dot{1}}=-\frac{3}{4}, & \left(\sigma_{333}\right)^{2, \dot{2}}=\frac{3}{4}, \\
\left(\sigma_{011}\right)^{1, \dot{1}}=-\frac{1}{4}, & \left(\sigma_{011}\right)^{2, \dot{2}}=-\frac{1}{4}, \\
\left(\sigma_{022}\right)^{1, \dot{1}}=-\frac{1}{4}, & \left(\sigma_{022}\right)^{2, \dot{2}}=-\frac{1}{4}, \\
\left(\sigma_{033}\right)^{1, \dot{1}}=-\frac{1}{4}, & \left(\sigma_{033}\right)^{2, \dot{2}}=-\frac{1}{4}, \\
\left(\sigma_{033}\right)^{1, \dot{1}}=-\frac{1}{4}, & \left(\sigma_{033}\right)^{2, \dot{2}}=-\frac{1}{4}, \\
\left(\sigma_{122}\right)^{1, \dot{2}}=-\frac{1}{4}, & \left(\sigma_{122}\right)^{2, \dot{1}}=-\frac{1}{4}, \\
\left(\sigma_{133}\right)^{1, \dot{2}}=-\frac{1}{4}, & \left(\sigma_{133}\right)^{2, \dot{1}}=-\frac{1}{4}, \\
\sigma^{1, \dot{2}}=-\frac{i}{4}, & \left(\sigma_{233}\right)^{2, \dot{1}}=-\frac{i}{4} .
\end{array}
$$

\section{Acknowledgements}

I would like to acknowledge G. Moultaka and M. Rausch de Traubenberg for their important help. I would also like to thank R. Kerner for very useful remarks.

[1] O'Raifeartaigh L., Mass differences and Lie algebras of finite order, Phys. Rev. Lett., 1965, V.14, 575-577.

[2] Coleman S., Mandula J., All possible symmetries of the $S$ matrix, Phys. Rev., 1967, V.159, 1251-1256.

[3] Lopuszanski J., An introduction to symmetry and supersymmetry in quantum field theory, Singapore, World Scientific Publishing, 1991.

[4] Streater R.F., Wightman A.S., PCT, spin and statistics and all that, New York, W.A. Benjamin, Inc., 1964.

[5] Haag R., Lopuszanski J.T., Sohnius M.F., All possible generators of supersymmetries of the $S$ matrix, Nucl. Phys. B, 1976, V.88, 257-274.

[6] Rausch de Traubenberg M., Slupinski M.J., Finite-dimensional Lie algebras of order F, J. Math. Phys., 2002, V.43, 5145-5160, hep-th/0205113.

[7] Rausch de Traubenberg M., Slupinski M.J., Fractional supersymmetry and $F($ th) roots of representations, J. Math. Phys., 2000, V.41, 4556-4571, hep-th/9904126.

[8] Mohammedi N., Moultaka G., Rausch de Traubenberg M., Field theoretic realizations for cubic supersymmetry, Internat. J. Modern Phys. A, 2004, V.19, 5585-5608, hep-th/0305172.

[9] Moultaka G., Rausch de Traubenberg M., Tanasa A., Cubic supersymmetry and Abelian gauge invariance, Internat. J. Modern Phys. A, 2005, V.20, 5779-5806, hep-th/0411198.

[10] Beckers J., Debergh N., On parasupersymmetric coherent states, Modern Phys. Lett. A, 1989, V.4, 12091215.

[11] Beckers J., Debergh N., Poincaré invariance and quantum parasuperfields, Internat. J. Modern Phys. A, 1993, V.8, 5041-5061.

[12] Rubakov V.A., Spiridonov V.P., Parasupersymmetric quantum mechanics, Modern Phys. Lett. A, 1988, V.3, 1337-1347. 
[13] Matheus-Valle J.L., Monteiro M.A.R., Quantum group generalization of the classical supersymmetric point particle, Modern Phys. Lett. A, 1992, V.7, 3023-3028.

[14] Durand S., Extended fractional supersymmetric quantum mechanics, Modern Phys. Lett. A, 1993, V.8, 1795-1804, hep-th/9305130.

[15] de Azcàrraga J.A., Macfarlane A.J., Group theoretical foundations of fractional supersymmetry, J. Math. Phys., 1996, V.37, 1115-1127, hep-th/9506177.

[16] Fleury N., Rausch de Traubenberg M., Local fractional supersymmetry for alternative statistics, Modern Phys. Lett. A, 1996, V.11, 899-914, hep-th/9510108.

[17] Dunne R.S., Macfarlane A.J., de Azcarraga J.A., Perez Bueno J.C., Geometrical foundations of fractional supersymmetry, Internat. J. Modern Phys. A, 1997, V.12, 3275-3306, hep-th/9610087.

[18] Perez A., Rausch de Traubenberg M., Simon P., 2D fractional supersymmetry for rational conformal field theory. Application for third-integer spin states, Nucl. Phys. B, 1996, V.482, 325-344, hep-th/9603149.

[19] Rausch de Traubenberg M., Simon P., 2-D fractional supersymmetry and conformal field theory for alternative statistics, Nucl. Phys. B, 1998, V.517, 485-505, hep-th/9606188.

[20] Rausch de Traubenberg M., Slupinski M.J., Non-trivial extensions of the 3D-Poincaré algebra and fractional supersymmetry for anyons, Modern Phys. Lett. A, 1997, V.12, 3051-3066, hep-th/9609203.

[21] Kerner R., $Z(3)$ graded algebras and the cubic root of the supersymmetry translations, J. Math. Phys., 1992, V.33, 403-411.

[22] Filippov V.T., n-Lie algebras, Sibirsk. Mat. Zh., 1985, V.26, 126-140.

[23] Tanasa A., Lie subalgebras of the Weyl algebra. Lie algebras of order 3 and their application to cubic supersymmetry, hep-th/0509174.

[24] Goze M., Rausch de Traubenberg M., Tanasa A., Deformations, contractions and classifications of Lie algebras of order 3, math-ph/0603008.

[25] Sohnius M.F., Introducing supersymmetry, Phys. Rep., 1985, V.128, 39-204.

[26] Grignani G., Plyushchay M., Sodano P., A pseudoclassical model for P,T-invariant planar fermions, Nucl. Phys. B, 1996, V.464, 189-212, hep-th/9511072.

[27] Nirov K.S., Plyushchay M.S., P,T-invariant system of Chern-Simons fields: pseudoclassical model and hidden symmetries, Nucl. Phys. B, 1998, V.512, 295-319, hep-th/9803221.

[28] Plyushchay M.S., Hidden nonlinear supersymmetries in pure parabosonic systems, Internat. J. Modern Phys. A, 2000, V.15, 3679-3698, hep-th/9903130.

[29] Plyushchay M.S., Rausch de Traubenberg M., Cubic root of Klein-Gordon equation, Phys. Lett. B, 2000, V.477, 276-284, hep-th/0001067.

[30] Witten E., Lecture notes on supersymmetry, in Proc. Inter. School of Subnuclear Physics (Erice, 1981), Editor A. Zichichi, Plenum Press, 1983, 1-64.

[31] Polchinski J., String theory, Cambridge University Press, 2000.

[32] Gomis J., Paris J., Samuel S., Antibracket, antifields and gauge-theory quantization, Phys. Rep., 1995, V.259, 1-145, hep-th/9412228.

[33] Peskin M., Schroeder D., An introduction to quantum field theory, Perseus Books, 1980

[34] Horowitz G.T., Exactly soluble diffeomorphism invariant theories, Comm. Math. Phys., 1989, V.125, 417437.

[35] Horowitz G.T., Srednicki M., A quantum field theoretic description of linking numbers and their generalization, Comm. Math. Phys., 1990, V.130, 83-94.

[36] Blau M., Thompson G., Topological gauge theories of antisymmetric tensor fields, Ann. Phys., 1991, V.205, 130-172.

[37] Blau M., Thompson G., Do metric independent classical actions lead to topological field theories?, Phys. Lett. B, 1991, V.255, 535-542.

[38] Rausch de Traubenberg M., Four dimensional cubic supersymmetry, hep-th/0312066.

[39] Moultaka G., Rausch de Traubenberg M., Tanasa A., Non-trivial extension of the Poincaré algebra for antisymmetric gauge fields, Contribution to the XI-th International Conference "Symmetry Methods in Physics" (June 21-24, 2004, Prague), hep-th/0407168.

[40] Henneaux M., All consistent interactions for exterior form gauge fields, Phys. Rev. D, 1997, V.56, 6076-6080, hep-th/9706119. 